\title{
Erratum: Search for new non-resonant phenomena in high-mass dilepton final states with the ATLAS detector
}

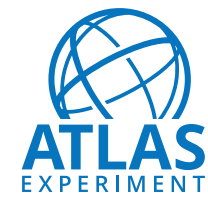

\section{The ATLAS collaboration}

E-mail: atlas.publications@cern.ch

ERratum TO: JHEP11(2020)005

ArXiv EPrint: 2006.12946

The Lagrangian in eq. (2.1) of JHEP 11 (2020) 005 has an erroneous factor of $\frac{1}{2}$. The analysis code used for the results reported in the paper does not have this factor. The results remain unchanged.

$$
\begin{aligned}
\mathcal{L}=\frac{g^{2}}{\Lambda^{2}}\{ & \eta_{\mathrm{LL}}\left(\bar{q}_{\mathrm{L}} \gamma_{\mu} q_{\mathrm{L}}\right)\left(\bar{\ell}_{\mathrm{L}} \gamma^{\mu} \ell_{\mathrm{L}}\right) \\
& +\eta_{\mathrm{RR}}\left(\bar{q}_{\mathrm{R}} \gamma_{\mu} q_{\mathrm{R}}\right)\left(\bar{\ell}_{\mathrm{R}} \gamma^{\mu} \ell_{\mathrm{R}}\right) \\
& +\eta_{\mathrm{LR}}\left(\bar{q}_{\mathrm{L}} \gamma_{\mu} q_{\mathrm{L}}\right)\left(\bar{\ell}_{\mathrm{R}} \gamma^{\mu} \ell_{\mathrm{R}}\right) \\
& \left.+\eta_{\mathrm{RL}}\left(\bar{q}_{\mathrm{R}} \gamma_{\mu} q_{\mathrm{R}}\right)\left(\bar{\ell}_{\mathrm{L}} \gamma^{\mu} \ell_{\mathrm{L}}\right)\right\},
\end{aligned}
$$

Open Access. This article is distributed under the terms of the Creative Commons Attribution License (CC-BY 4.0), which permits any use, distribution and reproduction in any medium, provided the original author(s) and source are credited. 


\section{The ATLAS collaboration}

G. $\operatorname{Aad}^{102}$, B. Abbott ${ }^{128}$, D.C. Abbott ${ }^{103}$, A. Abed Abud ${ }^{36}$, K. Abeling ${ }^{53}$, D.K. Abhayasinghe ${ }^{94}$, S.H. Abidi ${ }^{166}$, O.S. AbouZeid ${ }^{40}$, N.L. Abraham ${ }^{155}$,

H. Abramowicz ${ }^{160}$, H. Abreu ${ }^{159}$, Y. Abulaiti ${ }^{6}$, B.S. Acharya ${ }^{67 a, 67 b, n}$, B. Achkar ${ }^{53}$,

L. $\operatorname{Adam}^{100}$, C. Adam Bourdarios 5 , L. Adamczyk ${ }^{84 a}$, L. Adamek ${ }^{166}$, J. Adelman ${ }^{121}$,

M. Adersberger ${ }^{114}$, A. Adiguzel ${ }^{12 c}$, S. Adorni ${ }^{54}$, T. Adye ${ }^{143}$, A.A. Affolder ${ }^{145}$, Y. Afik ${ }^{159}$,

C. Agapopoulou ${ }^{65}$, M.N. Agaras ${ }^{38}$, A. Aggarwal ${ }^{119}$, C. Agheorghiesei ${ }^{27 c}$,

J.A. Aguilar-Saavedra ${ }^{139 f, 139 a, a d}$, A. Ahmad ${ }^{36}$, F. Ahmadov ${ }^{80}$, W.S. Ahmed ${ }^{104}$, X. Ai $^{18}$,

G. Aielli ${ }^{74 a, 74 b}$, S. Akatsuka ${ }^{86}$, T.P.A. Åkesson ${ }^{97}$, E. Akilli ${ }^{54}$, A.V. Akimov ${ }^{111}$,

K. Al Khoury ${ }^{65}$, G.L. Alberghi ${ }^{23 b, 23 a}$, J. Albert ${ }^{175}$, M.J. Alconada Verzini ${ }^{160}$,

S. Alderweireldt ${ }^{36}$, M. Aleksa ${ }^{36}$, I.N. Aleksandrov ${ }^{80}$, C. Alexa ${ }^{27 b}$, T. Alexopoulos ${ }^{10}$,

A. Alfonsi ${ }^{120}$, F. Alfonsi ${ }^{23 b, 23 a}$, M. Alhroob ${ }^{128}$, B. Ali ${ }^{141}$, S. Ali ${ }^{157}$, M. Aliev ${ }^{165}$,

G. Alimonti ${ }^{69 a}$, C. Allaire ${ }^{36}$, B.M.M. Allbrooke ${ }^{155}$, B.W. Allen ${ }^{131}$, P.P. Allport ${ }^{21}$,

A. Aloisio $^{70 a, 70 b}$, F. Alonso ${ }^{89}$, C. Alpigiani ${ }^{147}$, E. Alunno Camelia ${ }^{74 a, 74 b}$,

M. Alvarez Estevez ${ }^{99}$, M.G. Alviggi ${ }^{70 a, 70 b}$, Y. Amaral Coutinho ${ }^{81 b}$, A. Ambler ${ }^{104}$,

L. Ambroz ${ }^{134}$, C. Amelung ${ }^{26}$, D. Amidei ${ }^{106}$, S.P. Amor Dos Santos ${ }^{139 a}$, S. Amoroso ${ }^{46}$,

C.S. Amrouche ${ }^{54}$, F. An ${ }^{79}$, C. Anastopoulos ${ }^{148}$, N. Andari ${ }^{144}$, T. Andeen ${ }^{11}$,

J.K. Anders ${ }^{20}$, S.Y. Andrean ${ }^{45 a, 45 b}$, A. Andreazza ${ }^{69 a, 69 b}$, V. Andrei ${ }^{61 a}$, C.R. Anelli ${ }^{175}$,

S. Angelidakis ${ }^{9}$, A. Angerami ${ }^{39}$, A.V. Anisenkov ${ }^{122 b, 122 a}$, A. Annovi ${ }^{72 a}$, C. $^{2}$ Antel $^{54}$,

M.T. Anthony ${ }^{148}$, E. Antipov ${ }^{129}$, M. Antonelli ${ }^{51}$, D.J.A. Antrim ${ }^{170}$, F. Anulli ${ }^{73 a}$,

M. Aoki ${ }^{82}$, J.A. Aparisi Pozo ${ }^{173}$, M.A. Aparo ${ }^{155}$, L. Aperio Bella ${ }^{46}$,

N. Aranzabal Barrio ${ }^{36}$, V. Araujo Ferraz ${ }^{81 a}$, R. Araujo Pereira ${ }^{81 b}$, C. Arcangeletti ${ }^{51}$,

A.T.H. Arce ${ }^{49}$, F.A. Arduh ${ }^{89}$, J-F. Arguin ${ }^{110}$, S. Argyropoulos ${ }^{52}$, J.-H. Arling ${ }^{46}$,

A.J. Armbruster ${ }^{36}$, A. Armstrong ${ }^{170}$, O. Arnaez ${ }^{166}$, H. Arnold ${ }^{120}$,

Z.P. Arrubarrena Tame ${ }^{114}$, G. Artoni ${ }^{134}$, K. Asai ${ }^{126}$, S. Asai ${ }^{162}$, T. Asawatavonvanich ${ }^{164}$,

N. Asbah ${ }^{59}$, E.M. Asimakopoulou ${ }^{171}$, L. Asquith ${ }^{155}$, J. Assahsah ${ }^{35 d}$, K. Assamagan ${ }^{29}$,

R. Astalos ${ }^{28 a}$, R.J. Atkin ${ }^{33 a}$, M. Atkinson ${ }^{172}$, N.B. Atlay ${ }^{19}$, H. Atmani ${ }^{65}$, K. Augsten ${ }^{141}$,

V.A. Austrup ${ }^{181}$, G. Avolio ${ }^{36}$, M.K. Ayoub ${ }^{15 a}$, G. Azuelos ${ }^{110, a l}$, H. Bachacou ${ }^{144}$,

K. Bachas ${ }^{161}$, M. Backes ${ }^{134}$, F. Backman ${ }^{45 a, 45 b}$, P. Bagnaia ${ }^{73 a, 73 b}$, M. Bahmani ${ }^{85}$,

H. Bahrasemani ${ }^{151}$, A.J. Bailey ${ }^{173}$, V.R. Bailey ${ }^{172}$, J.T. Baines ${ }^{143}$, C. Bakalis ${ }^{10}$,

O.K. Baker ${ }^{182}$, P.J. Bakker ${ }^{120}$, E. Bakos ${ }^{16}$, D. Bakshi Gupta ${ }^{8}$, S. Balaji ${ }^{156}$,

E.M. Baldin ${ }^{122 b, 122 a}$, P. Balek ${ }^{179}$, F. Balli ${ }^{144}$, W.K. Balunas ${ }^{134}$, J. Balz ${ }^{100}$, E. Banas ${ }^{85}$,

M. Bandieramonte ${ }^{138}$, A. Bandyopadhyay ${ }^{24}$, Sw. Banerjee ${ }^{180, \mathrm{i}}$, L. Barak $^{160}$,

W.M. Barbe ${ }^{38}$, E.L. Barberio ${ }^{105}$, D. Barberis ${ }^{55 b, 55 a}$, M. Barbero ${ }^{102}$, G. Barbour ${ }^{95}$,

T. Barillari ${ }^{115}$, M-S. Barisits ${ }^{36}$, J. Barkeloo ${ }^{131}$, T. Barklow ${ }^{152}$, R. Barnea ${ }^{159}$,

B.M. Barnett ${ }^{143}$, R.M. Barnett ${ }^{18}$, Z. Barnovska-Blenessy ${ }^{60 a}$, A. Baroncelli ${ }^{60 a}$,

G. Barone ${ }^{29}$, A.J. Barr ${ }^{134}$, L. Barranco Navarro ${ }^{45 a, 45 b}$, F. Barreiro ${ }^{99}$,

J. Barreiro Guimarães da Costa ${ }^{15 a}$, U. Barron ${ }^{160}$, S. Barsov ${ }^{137}$, F. Bartels ${ }^{61 a}$,

R. Bartoldus ${ }^{152}$, G. Bartolini ${ }^{102}$, A.E. Barton ${ }^{90}$, P. Bartos $^{28 a}$, A. Basalaev ${ }^{46}$, A. Basan ${ }^{100}$,

A. Bassalat ${ }^{65, \text { ai }}$, M.J. Basso ${ }^{166}$, R.L. Bates ${ }^{57}$, S. Batlamous ${ }^{35 e}$, J.R. Batley ${ }^{32}$,

B. Batool ${ }^{150}$, M. Battaglia ${ }^{145}$, M. Bauce ${ }^{73 a}, 73 b$, F. Bauer ${ }^{144}$, K.T. Bauer ${ }^{170}$, H.S. Bawa ${ }^{31}$,

A. Bayirli ${ }^{12 c}$, J.B. Beacham ${ }^{49}$, T. Beau ${ }^{135}$, P.H. Beauchemin ${ }^{169}$, F. Becherer ${ }^{52}$, 
P. Bechtle ${ }^{24}$, H.C. Beck ${ }^{53}$, H.P. Beck ${ }^{20, p}$, K. Becker ${ }^{177}$, C. Becot ${ }^{46}$, A. Beddall ${ }^{12 d}$, A.J. Beddall ${ }^{12 a}$, V.A. Bednyakov ${ }^{80}$, M. Bedognetti ${ }^{120}$, C.P. Bee ${ }^{154}$, T.A. Beermann ${ }^{181}$, M. Begalli ${ }^{81 b}$, M. Begel ${ }^{29}$, A. Behera ${ }^{154}$, J.K. Behr ${ }^{46}$, F. Beisiegel ${ }^{24}$, M. Belfkir ${ }^{5}$, A.S. Bell ${ }^{95}$, G. Bella ${ }^{160}$, L. Bellagamba ${ }^{23 b}$, A. Bellerive ${ }^{34}$, P. Bellos ${ }^{9}$,

K. Beloborodov ${ }^{122 b, 122 a}$, K. Belotskiy ${ }^{112}$, N.L. Belyaev ${ }^{112}$, D. Benchekroun ${ }^{35 a}$,

N. Benekos ${ }^{10}$, Y. Benhammou ${ }^{160}$, D.P. Benjamin ${ }^{6}$, M. Benoit $^{54}$, J.R. Bensinger ${ }^{26}$,

S. Bentvelsen ${ }^{120}$, L. Beresford ${ }^{134}$, M. Beretta ${ }^{51}$, D. Berge ${ }^{19}$, E. Bergeaas Kuutmann ${ }^{171}$,

N. Berger ${ }^{5}$, B. Bergmann ${ }^{141}$, L.J. Bergsten ${ }^{26}$, J. Beringer ${ }^{18}$, S. Berlendis ${ }^{7}$, G. Bernardi ${ }^{135}$, C. Bernius ${ }^{152}$, F.U. Bernlochner ${ }^{24}$, T. Berry ${ }^{94}$, P. Berta ${ }^{100}$, C. Bertella ${ }^{15 a}$, A. Berthold ${ }^{48}$, I.A. Bertram ${ }^{90}$, O. Bessidskaia Bylund ${ }^{181}$, N. Besson ${ }^{144}$, A. Bethani ${ }^{101}$, S. Bethke ${ }^{115}$, A. Betti ${ }^{42}$, A.J. Bevan ${ }^{93}$, J. Beyer ${ }^{115}$, D.S. Bhattacharya ${ }^{176}$, P. Bhattarai ${ }^{26}$, V.S. Bhopatkar ${ }^{6}$, R. Bi ${ }^{138}$, R.M. Bianchi ${ }^{138}$, O. Biebel ${ }^{114}$, D. Biedermann ${ }^{19}$, R. Bielski ${ }^{36}$, K. Bierwagen ${ }^{100}$, N.V. Biesuz ${ }^{72 a, 72 b}$, M. Biglietti ${ }^{75 a}$, T.R.V. Billoud ${ }^{110}$, M. Bindi $^{53}$, A. Bingul $^{12 d}$, C. Bini ${ }^{73 a, 73 b}$, S. Biondi ${ }^{23 b, 23 a}$, C.J. Birch-sykes ${ }^{101}$, M. Birman ${ }^{179}$, T. Bisanz ${ }^{53}$, J.P. Biswal ${ }^{3}$, D. Biswas ${ }^{180, i}$, A. Bitadze ${ }^{101}$, C. Bittrich ${ }^{48}$, K. Bjørke $^{133}$, T. Blazek ${ }^{28 a}$, I. Bloch ${ }^{46}$, C. Blocker ${ }^{26}$, A. Blue ${ }^{57}$, U. Blumenschein ${ }^{93}$, G.J. Bobbink ${ }^{120}$, V.S. Bobrovnikov ${ }^{122 b, 122 a}$, S.S. Bocchetta ${ }^{97}$, D. Bogavac ${ }^{14}$, A.G. Bogdanchikov ${ }^{122 b, 122 a}$, C. Bohm ${ }^{45 a}$, V. Boisvert ${ }^{94}$, P. Bokan ${ }^{53,171,53}$, T. Bold ${ }^{84 a}$, A.E. Bolz ${ }^{61 b}$, M. Bomben ${ }^{135}$, M. Bona ${ }^{93}$, J.S. Bonilla ${ }^{131}$, M. Boonekamp ${ }^{144}$, C.D. Booth ${ }^{94}$, H.M. Borecka-Bielska ${ }^{91}$, L.S. Borgna ${ }^{95}$, A. Borisov ${ }^{123}$, G. Borissov ${ }^{90}$, J. Bortfeldt ${ }^{36}$, D. Bortoletto ${ }^{134}$, D. Boscherini2 ${ }^{23 b}$, M. Bosman ${ }^{14}$, J.D. Bossio Sola ${ }^{104}$, K. Bouaouda ${ }^{35 a}$, J. Boudreau ${ }^{138}$, E.V. Bouhova-Thacker ${ }^{90}$, D. Boumediene ${ }^{38}$, S.K. Boutle ${ }^{57}$, A. Boveia ${ }^{127}$, J. Boyd ${ }^{36}$, D. Boye ${ }^{33 c}$, I.R. Boyko ${ }^{80}$, A.J. Bozson ${ }^{94}$, J. Bracinik ${ }^{21}$, N. Brahimi ${ }^{60 d}$, G. Brandt ${ }^{181}$, O. Brandt ${ }^{32}$, F. Braren ${ }^{46}$, B. Brau ${ }^{103}$, J.E. Brau ${ }^{131}$, W.D. Breaden Madden ${ }^{57}$, K. Brendlinger ${ }^{46}$, L. Brenner ${ }^{36}$, R. Brenner ${ }^{171}$, S. Bressler ${ }^{179}$, B. Brickwedde ${ }^{100}$, D.L. Briglin ${ }^{21}$, D. Britton ${ }^{57}$, D. Britzger ${ }^{115}$, I. Brock ${ }^{24}$, R. Brock ${ }^{107}$, G. Brooijmans ${ }^{39}$, W.K. Brooks ${ }^{146 d}$, E. Brost ${ }^{29}$, P.A. Bruckman de Renstrom ${ }^{85}$, B. Brüers ${ }^{46}$, D. Bruncko ${ }^{28 b}$, A. Bruni ${ }^{23 b}$, G. Bruni ${ }^{23 b}$, L.S. Bruni ${ }^{120}$, S. Bruno ${ }^{74 a, 74 b}$, M. Bruschi ${ }^{23 b}$, N. Bruscino ${ }^{73 a}, 73 b$, L. Bryngemark ${ }^{152}$, T. Buanes ${ }^{17}$, Q. Buat ${ }^{36}$, P. Buchholz ${ }^{150}$, A.G. Buckley ${ }^{57}$, I.A. Budagov ${ }^{80}$, M.K. Bugge ${ }^{133}$, F. Bührer ${ }^{52}$, O. Bulekov ${ }^{112}$, B.A. Bullard ${ }^{59}$, T.J. Burch ${ }^{121}$, S. Burdin ${ }^{91}$, C.D. Burgard ${ }^{120}$, A.M. Burger ${ }^{129}$, B. Burghgrave ${ }^{8}$, J.T.P. Burr ${ }^{46}$, C.D. Burton ${ }^{11}$, J.C. Burzynski1 ${ }^{103}$, V. Büscher ${ }^{100}$, E. Buschmann ${ }^{53}$, P.J. Bussey ${ }^{57}$, J.M. Butler ${ }^{25}$, C.M. Buttar ${ }^{57}$, J.M. Butterworth ${ }^{95}$, P. Butti ${ }^{36}$, W. Buttinger ${ }^{36}$, C.J. Buxo Vazquez ${ }^{107}$, A. Buzatu ${ }^{157}$, A.R. Buzykaev ${ }^{122 b, 122 a}$, G. Cabras ${ }^{23 b}$,23a, S. Cabrera Urbán ${ }^{173}$, D. Caforio ${ }^{56}$, H. Cai ${ }^{138}$, V.M.M. Cairo ${ }^{152}$, O. Cakir $^{4 a}$, N. Calace $^{36}$, P. Calafiura ${ }^{18}$, G. Calderini ${ }^{135}$, P. Calfayan ${ }^{66}$, G. Callea ${ }^{57}$, L.P. Caloba ${ }^{81 b}$, A. Caltabiano ${ }^{74 a, 74 b}$, S. Calvente Lopez $^{99}$, D. Calvet ${ }^{38}$, S. Calvet ${ }^{38}$, T.P. Calvet ${ }^{102}$, M. Calvetti ${ }^{72 a, 72 b}$, R. Camacho Toro ${ }^{135}$, S. Camarda ${ }^{36}$,

D. Camarero Munoz ${ }^{99}$, P. Camarri ${ }^{74 a, 74 b}$, M.T. Camerlingo ${ }^{75 a, 75 b}$, D. Cameron ${ }^{133}$,

C. Camincher $^{36}$, S. Campana ${ }^{36}$, M. Campanelli ${ }^{95}$, A. Camplani ${ }^{40}$, V. Canale ${ }^{70 a}, 70 b$,

A. Canesse ${ }^{104}$, M. Cano Bret ${ }^{78}$, J. Cantero ${ }^{129}$, T. Cao ${ }^{160}$, Y. Cao ${ }^{91}$, M.D.M. Capeans Garrido ${ }^{36}$, M. Capua ${ }^{41 b, 41 a}$, R. Cardarelli ${ }^{74 a}$, F. Cardillo ${ }^{148}$, G. Carducci ${ }^{41 b, 41 a}$, I. Carli ${ }^{142}$, T. Carli ${ }^{36}$, G. Carlino ${ }^{70 a}$, B.T. Carlson ${ }^{138}$, 
E.M. Carlson ${ }^{175,167 a}$, L. Carminati ${ }^{69 a, 69 b}$, R.M.D. Carney ${ }^{152}$, S. Caron ${ }^{119}$, E. Carquin ${ }^{146 d}$, S. Carrá ${ }^{46}$, G. Carratta ${ }^{23 b, 23 a}$, J.W.S. Carter ${ }^{166}$, T.M. Carter ${ }^{50}$, M.P. Casado ${ }^{14, f}$, A.F. Casha ${ }^{166}$, F.L. Castillo ${ }^{173}$, L. Castillo Garcia ${ }^{14}$, V. Castillo Gimenez ${ }^{173}$, N.F. Castro ${ }^{139 a, 139 e}$, A. Catinaccio ${ }^{36}$, J.R. Catmore ${ }^{133}$, A. Cattai ${ }^{36}$, V. Cavaliere $^{29}$, V. Cavasinni ${ }^{72 a}, 72 b$, E. Celebi ${ }^{12 b}$, F. Celli ${ }^{134}$, K. Cerny ${ }^{130}$, A.S. Cerqueira ${ }^{81 a}$, A. Cerri ${ }^{155}$, L. Cerrito ${ }^{74 a, 74 b}$, F. Cerutti ${ }^{18}$, A. Cervelli23b,23a , S.A. Cetin ${ }^{12 b}$, Z. Chadi ${ }^{35 a}$, D. Chakraborty ${ }^{121}$, J. Chan ${ }^{180}$, W.S. Chan ${ }^{120}$, W.Y. Chan ${ }^{91}$, J.D. Chapman ${ }^{32}$, B. Chargeishvili ${ }^{158 b}$, D.G. Charlton ${ }^{21}$, T.P. Charman $^{93}$, C.C. $\mathrm{Chau}^{34}$, S. Che ${ }^{127}$, S. Chekanov ${ }^{6}$, S.V. Chekulaev ${ }^{167 a}$, G.A. Chelkov ${ }^{80, a g}$, B. Chen ${ }^{79}$, C. Chen ${ }^{60 a}$, C.H. Chen ${ }^{79}$, H. Chen ${ }^{29}$, J. Chen ${ }^{60 a}$, J. Chen ${ }^{39}$, J. Chen ${ }^{26}$, S. Chen ${ }^{136}$, S.J. Chen ${ }^{15 c}$, X. Chen ${ }^{15 b}$, Y. Chen ${ }^{60 a}$, Y-H. Chen ${ }^{46}$, H.C. Cheng ${ }^{63 a}$, H.J. Cheng ${ }^{15 a}$, A. Cheplakov ${ }^{80}$, E. Cheremushkina ${ }^{123}$, R. Cherkaoui El Moursli ${ }^{35 e}$, E. Cheu ${ }^{7}$, K. Cheung ${ }^{64}$, T.J.A. Chevalérias ${ }^{144}$, L. Chevalier ${ }^{144}$, V. Chiarella ${ }^{51}$, G. Chiarelli ${ }^{72 a}$, G. Chiodini ${ }^{68 a}$, A.S. Chisholm ${ }^{21}$, A. Chitan ${ }^{27 b}$, I. Chiu ${ }^{162}$, Y.H. Chiu ${ }^{175}$, M.V. Chizhov ${ }^{80}$, K. Choi $^{11}$, A.R. Chomont ${ }^{73 a, 73 b}$, Y.S. Chow ${ }^{120}$, L.D. Christopher ${ }^{33 e}$, M.C. Chu ${ }^{63 a}$, X. Chu ${ }^{15 a, 15 d}$, J. Chudoba ${ }^{140}$, J.J. Chwastowski ${ }^{85}$, L. Chytka ${ }^{130}$, D. Cieri ${ }^{115}$, K.M. Ciesla ${ }^{85}$, D. Cinca ${ }^{47}$,

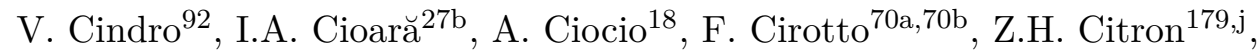
M. Citterio ${ }^{69 a}$, D.A. Ciubotaru ${ }^{27 b}$, B.M. Ciungu ${ }^{166}$, A. Clark ${ }^{54}$, M.R. Clark ${ }^{39}$, P.J. Clark ${ }^{50}$, S.E. Clawson ${ }^{101}$, C. Clement ${ }^{45 a, 45 b}$, Y. Coadou ${ }^{102}$, M. Cobal ${ }^{67 a, 67 c}$, A. Coccaro ${ }^{55 b}$, J. Cochran ${ }^{79}$, R. Coelho Lopes De $\mathrm{Sa}^{103}$, H. Cohen ${ }^{160}$, A.E.C. Coimbra ${ }^{36}$, B. Cole $^{39}$, A.P. Colijn ${ }^{120}$, J. Collot ${ }^{58}$, P. Conde Muiño ${ }^{139 a, 139 h}$, S.H. Connell ${ }^{33 c}$, I.A. Connelly ${ }^{57}$, S. Constantinescu ${ }^{27 b}$, F. Conventi ${ }^{70 a, a m}$, A.M. Cooper-Sarkar ${ }^{134}$, F. Cormier ${ }^{174}$, K.J.R. Cormier ${ }^{166}$, L.D. Corpe ${ }^{95}$, M. Corradi ${ }^{73 a, 73 b}$, E.E. Corrigan ${ }^{97}$, F. Corriveau ${ }^{104, a b}$, M.J. Costa ${ }^{173}$, F. Costanza ${ }^{5}$, D. Costanzo ${ }^{148}$, G. Cowan ${ }^{94}$, J.W. Cowley ${ }^{32}$, J. Crane ${ }^{101}$, K. Cranmer ${ }^{125}$, R.A. Creager ${ }^{136}$, S. Crépé-Renaudin ${ }^{58}$, F. Crescioli ${ }^{135}$, M. Cristinziani ${ }^{24}$, V. Croft ${ }^{169}$, G. Crosetti ${ }^{41 \mathrm{~b}, 41 \mathrm{a}}$, A. Cueto ${ }^{5}$, T. Cuhadar Donszelmann ${ }^{170}$, H. Cui ${ }^{15 a, 15 d}$, A.R. Cukierman ${ }^{152}$, W.R. Cunningham ${ }^{57}$, S. Czekierda ${ }^{85}$, P. Czodrowski ${ }^{36}$, M.M. Czurylo ${ }^{61 b}$, M.J. Da Cunha Sargedas De Sousa ${ }^{60 b}$, J.V. Da Fonseca Pinto ${ }^{81 b}$, C. Da Via ${ }^{101}$, W. Dabrowski ${ }^{84 a}$, F. Dachs ${ }^{36}$, T. Dado ${ }^{47}$, S. Dahbi ${ }^{33 e}$, T. Dai ${ }^{106}$, C. Dallapiccola ${ }^{103}$, M. Dam ${ }^{40}$, G. D'amen ${ }^{29}$, V. D'Amico ${ }^{75 a, 75 b}$, J. Damp ${ }^{100}$, J.R. Dandoy ${ }^{136}$, M.F. Daneri ${ }^{30}$, M. Danninger ${ }^{151}$, V. Dao ${ }^{36}$, G. Darbo ${ }^{55 b}$, O. Dartsi ${ }^{5}$, A. Dattagupta ${ }^{131}$, T. Daubney ${ }^{46}$, S. D'Auria ${ }^{69 a, 69 b}$, C. David ${ }^{167 b}$,

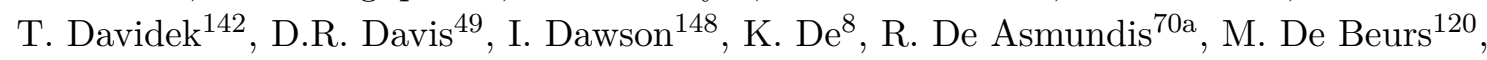
S. De Castro ${ }^{23 b, 23 a}$, N. De Groot ${ }^{119}$, P. de Jong ${ }^{120}$, H. De la Torre ${ }^{107}$, A. De Maria ${ }^{15 c}$, D. De Pedis ${ }^{73 a}$, A. De Salvo ${ }^{73 a}$, U. De Sanctis ${ }^{74 a, 74 b}$, M. De Santis ${ }^{74 a, 74 b}$, A. De Santo ${ }^{155}$, J.B. De Vivie De Regie ${ }^{65}$, C. Debenedetti ${ }^{145}$, D.V. Dedovich ${ }^{80}$, A.M. Deiana ${ }^{42}$,

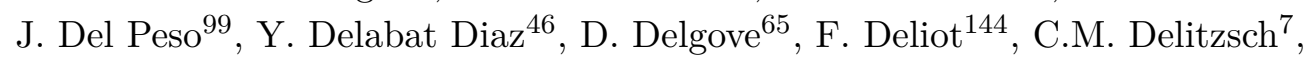
M. Della Pietra ${ }^{70 a, 70 b}$, D. Della Volpe ${ }^{54}$, A. Dell'Acqua ${ }^{36}$, L. Dell'Asta $^{74 a}, 74 b$, M. Delmastro ${ }^{5}$, C. Delporte ${ }^{65}$, P.A. Delsart ${ }^{58}$, D.A. DeMarco ${ }^{166}$, S. Demers ${ }^{182}$, M. Demichev ${ }^{80}$, G. Demontigny ${ }^{110}$, S.P. Denisov ${ }^{123}$, L. D'Eramo ${ }^{121}$, D. Derendarz ${ }^{85}$,

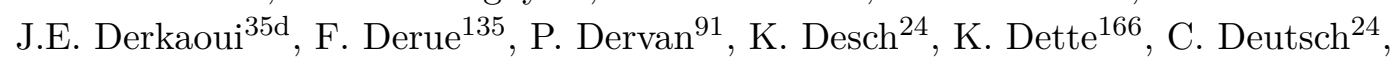
M.R. Devesa ${ }^{30}$, P.O. Deviveiros ${ }^{36}$, F.A. Di Bello ${ }^{73 a, 73 b}$, A. Di Ciaccio ${ }^{74 a, 74 b}$, L. Di Ciaccio ${ }^{5}$, W.K. Di Clemente ${ }^{136}$, C. Di Donato ${ }^{70 a, 70 b}$, A. Di Girolamo ${ }^{36}$, 
G. Di Gregorio ${ }^{72 a, 72 b}$, B. Di Micco ${ }^{75 a, 75 b}$, R. Di Nardo ${ }^{75 a, 75 b}$, K.F. Di Petrillo ${ }^{59}$, R. Di Sipio ${ }^{166}$, C. Diaconu ${ }^{102}$, F.A. Dias ${ }^{40}$, T. Dias Do Vale ${ }^{139 a}$, M.A. Diaz ${ }^{146 a}$, F.G. Diaz Capriles $^{24}$, J. Dickinson ${ }^{18}$, M. Didenko ${ }^{165}$, E.B. Diehl ${ }^{106}$, J. Dietrich ${ }^{19}$, S. Díez Cornell ${ }^{46}$, A. Dimitrievska ${ }^{18}$, W. Ding ${ }^{15 b}$, J. Dingfelder ${ }^{24}$, S.J. Dittmeier ${ }^{61 b}$, F. Dittus ${ }^{36}$, F. Djama ${ }^{102}$, T. Djobava ${ }^{158 b}$, J.I. Djuvsland ${ }^{17}$, M.A.B. Do Vale ${ }^{81 \mathrm{c}}$, M. Dobre ${ }^{27 b}$, D. Dodsworth ${ }^{26}$, C. Doglioni ${ }^{97}$, J. Dolejsi ${ }^{142}$, Z. Dolezal $^{142}$, M. Donadelli ${ }^{81 d}$,

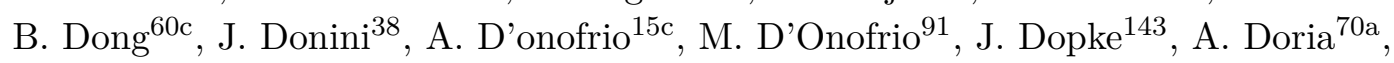
M.T. Dova ${ }^{89}$, A.T. Doyle ${ }^{57}$, E. Drechsler ${ }^{151}$, E. Dreyer ${ }^{151}$, T. Dreyer ${ }^{53}$, A.S. Drobac ${ }^{169}$, D. Du ${ }^{60 b}$, T.A. du Pree ${ }^{120}$, Y. Duan ${ }^{60 d}$, F. Dubinin ${ }^{111}$, M. Dubovsky ${ }^{28 a}$, A. Dubreuil ${ }^{54}$, E. Duchovni ${ }^{179}$, G. Duckeck ${ }^{114}$, O.A. Ducu ${ }^{36}$, D. Duda ${ }^{115}$, A. Dudarev ${ }^{36}$, A.C. Dudder ${ }^{100}$, E.M. Duffield ${ }^{18}$, M. D’uffizi ${ }^{101}$, L. Duflot ${ }^{65}$, M. Dührssen ${ }^{36}$, C. Dülsen ${ }^{181}$,

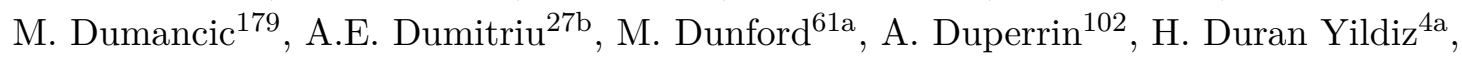
M. Düren ${ }^{56}$, A. Durglishvilii ${ }^{158 b}$, D. Duschinger ${ }^{48}$, B. Dutta ${ }^{46}$, D. Duvnjak ${ }^{1}$, G.I. Dyckes ${ }^{136}$, M. Dyndal ${ }^{36}$, S. Dysch ${ }^{101}$, B.S. Dziedzic ${ }^{85}$, M.G. Eggleston ${ }^{49}$, T. Eifert ${ }^{8}$, G. Eigen ${ }^{17}$, K. Einsweiler ${ }^{18}$, T. Ekelof ${ }^{171}$, H. El Jarrari ${ }^{35 e}$, V. Ellajosyula ${ }^{171}$, M. Ellert ${ }^{171}$, F. Ellinghaus ${ }^{181}$, A.A. Elliot ${ }^{93}$, N. Ellis ${ }^{36}$, J. Elmsheuser ${ }^{29}$, M. Elsing ${ }^{36}$, D. Emeliyanov ${ }^{143}$, A. Emerman ${ }^{39}$, Y. Enari ${ }^{162}$, M.B. Epland ${ }^{49}$, J. Erdmann ${ }^{47}$, A. Ereditato $^{20}$, P.A. Erland ${ }^{85}$, M. Errenst ${ }^{36}$, M. Escalier ${ }^{65}$, C. Escobar ${ }^{173}$, O. Estrada Pastor ${ }^{173}$, E. Etzion ${ }^{160}$, H. Evans ${ }^{66}$, M.O. Evans ${ }^{155}$, A. Ezhilov ${ }^{137}$, F. Fabbri ${ }^{57}$, L. Fabbri ${ }^{23 b, 23 a}$, V. Fabiani ${ }^{119}$, G. Facini ${ }^{177}$,

R.M. Faisca Rodrigues Pereira ${ }^{139 a}$, R.M. Fakhrutdinov ${ }^{123}$, S. Falciano ${ }^{73 a}$, P.J. Falke ${ }^{24}$, S. Falke ${ }^{36}$, J. Faltova ${ }^{142}$, Y. Fang ${ }^{15 a}$, Y. Fang ${ }^{15 a}$, G. Fanourakis ${ }^{44}$, M. Fanti ${ }^{69 a, 69 b}$, M. Faraj ${ }^{67 a, 67 c, q}$, A. Farbin ${ }^{8}$, A. Farilla ${ }^{75 a}$, E.M. Farina ${ }^{71 a, 71 b}$, T. Farooque ${ }^{107}$, S.M. Farrington ${ }^{50}$, P. Farthouat ${ }^{36}$, F. Fassi ${ }^{35 e}$, P. Fassnacht ${ }^{36}$, D. Fassouliotis ${ }^{9}$, M. Faucci Giannelli ${ }^{50}$, W.J. Fawcett ${ }^{32}$, L. Fayard ${ }^{65}$, O.L. Fedin ${ }^{137, o}$, W. Fedorko ${ }^{174}$, A. Fehr ${ }^{20}$, M. Feickert ${ }^{172}$, L. Feligioni ${ }^{102}$, A. Fell ${ }^{148}$, C. Feng ${ }^{60 b}$, M. Feng ${ }^{49}$, M.J. Fenton ${ }^{170}$, A.B. Fenyuk ${ }^{123}$, S.W. Ferguson ${ }^{43}$, J. Ferrando ${ }^{46}$, A. Ferrante ${ }^{172}$, A. Ferrari ${ }^{171}$, P. Ferrari ${ }^{120}$, R. Ferrari ${ }^{71 a}$, D.E. Ferreira de Lima ${ }^{61 b}$, A. Ferrer ${ }^{173}$, D. Ferrere ${ }^{54}$, C. Ferretti ${ }^{106}$, F. Fiedler ${ }^{100}$, A. Filipčic ${ }^{92}$, F. Filthaut ${ }^{119}$, K.D. Finelli ${ }^{25}$, M.C.N. Fiolhais ${ }^{139 a, 139 c, a}$, L. Fiorini ${ }^{173}$, F. Fischer ${ }^{114}$, J. Fischer ${ }^{100}$, W.C. Fisher ${ }^{107}$, T. Fitschen ${ }^{21}$, I. Fleck ${ }^{150}$, P. Fleischmann ${ }^{106}$, T. Flick ${ }^{181}$, B.M. Flierl ${ }^{114}$, L. Flores ${ }^{136}$, L.R. Flores Castillo ${ }^{63 a}$, F.M. Follega ${ }^{76 a, 76 b}$, N. Fomin ${ }^{17}$, J.H. Foo ${ }^{166}$, G.T. Forcolin ${ }^{76 a, 76 b}$, B.C. Forland ${ }^{66}$, A. Formica ${ }^{144}$, F.A. Förster ${ }^{14}$, A.C. Forti ${ }^{101}$, E. Fortin ${ }^{102}$, M.G. Foti ${ }^{134}$, D. Fournier ${ }^{65}$, H. Fox ${ }^{90}$, P. Francavilla ${ }^{72 a, 72 b}$, S. Francescato ${ }^{73 a, 73 b}$, M. Franchini ${ }^{23 b, 23 a}$, S. Franchino ${ }^{61 a}$, D. Francis ${ }^{36}$, L. Franco ${ }^{5}$, L. Franconi ${ }^{20}$, M. Franklin ${ }^{59}$, G. Frattari ${ }^{73 a, 73 b}$, A.N. Fray ${ }^{93}$, P.M. Freeman ${ }^{21}$, B. Freund ${ }^{110}$, W.S. Freund ${ }^{81 b}$, E.M. Freundlich ${ }^{47}$, D.C. Frizzell ${ }^{128}$, D. Froidevaux ${ }^{36}$, J.A. Frost ${ }^{134}$, M. Fujimoto ${ }^{126}$, C. Fukunaga ${ }^{163}$, E. Fullana Torregrosa ${ }^{173}$, T. Fusayasu ${ }^{116}$, J. Fuster ${ }^{173}$, A. Gabrielli ${ }^{23 b, 23 a}$, A. Gabrielli ${ }^{36}$, S. Gadatsch ${ }^{54}$, P. Gadow ${ }^{115}$, G. Gagliardi ${ }^{55 b, 55 a}$, L.G. Gagnon ${ }^{110}$, G.E. Gallardo ${ }^{134}$, E.J. Gallas ${ }^{134}$, B.J. Gallop ${ }^{143}$, G. Galster ${ }^{40}$, R. Gamboa Goni ${ }^{93}$, K.K. Gan ${ }^{127}$, S. Ganguly ${ }^{179}$, J. Gao ${ }^{60 a}$, Y. Gao ${ }^{50}$, Y.S. Gao ${ }^{31,1}$, F.M. Garay Walls ${ }^{146 a}$, C. García ${ }^{173}$, J.E. García Navarro ${ }^{173}$, J.A. García Pascual ${ }^{15 a}$, C. Garcia-Argos ${ }^{52}$, M. Garcia-Sciveres ${ }^{18}$, R.W. Gardner ${ }^{37}$, N. Garelli ${ }^{152}$, S. Gargiulo ${ }^{52}$, C.A. Garner ${ }^{166}$, V. Garonne ${ }^{133}$, 
S.J. Gasiorowski ${ }^{147}$, P. Gaspar ${ }^{81 b}$, A. Gaudiello ${ }^{55 b, 55 a}$, G. Gaudio ${ }^{71 a}$, I.L. Gavrilenko ${ }^{111}$, A. Gavrilyuk ${ }^{124}$, C. Gay ${ }^{174}$, G. Gaycken ${ }^{46}$, E.N. Gazis ${ }^{10}$, A.A. Geanta ${ }^{27 b}$, C.M. Gee ${ }^{145}$, C.N.P. Gee ${ }^{143}$, J. Geisen ${ }^{97}$, M. Geisen ${ }^{100}$, C. Gemme ${ }^{55 b}$, M.H. Genest ${ }^{58}$, C. Geng ${ }^{106}$, S. Gentile ${ }^{73 a}, 73 b$, S. George ${ }^{94}$, T. Geralis ${ }^{44}$, L.O. Gerlach ${ }^{53}$, P. Gessinger-Befurt ${ }^{100}$, G. Gessner ${ }^{47}$, S. Ghasemi ${ }^{150}$, M. Ghasemi Bostanabad ${ }^{175}$, M. Ghneimat ${ }^{150}$, A. Ghosh ${ }^{65}$, A. Ghosh ${ }^{78}$, B. Giacobbe ${ }^{23 b}$, S. Giagu ${ }^{73 a, 73 b}$, N. Giangiacomi ${ }^{23 b, 23 a}$, P. Giannetti ${ }^{72 a}$, A. Giannini ${ }^{70 a, 70 b}$, G. Giannini ${ }^{14}$, S.M. Gibson ${ }^{94}$, M. Gignac $^{145}$, D.T. Gil ${ }^{84 b}$, D. Gillberg ${ }^{34}$, G. Gilles ${ }^{181}$, D.M. Gingrich ${ }^{3, a l}$, M.P. Giordani ${ }^{67 a, 67 c}$, P.F. Giraud ${ }^{144}$, G. Giugliarelli ${ }^{67 a, 67 c}$, D. Giugni ${ }^{69 a}$, F. Giuli ${ }^{74 a, 74 b}$, S. Gkaitatzis ${ }^{161}$, I. Gkialas ${ }^{9, g}$, E.L. Gkougkousis ${ }^{14}$, P. Gkountoumis ${ }^{10}$, L.K. Gladilin ${ }^{113}$, C. Glasman ${ }^{99}$, J. Glatzer ${ }^{14}$, P.C.F. Glaysher ${ }^{46}$, A. Glazov ${ }^{46}$, G.R. Gledhill ${ }^{131}$, I. Gnesi ${ }^{41 b, b}$, M. Goblirsch-Kolb ${ }^{26}$, D. Godin ${ }^{110}$, S. Goldfarb ${ }^{105}$, T. Golling ${ }^{54}$, D. Golubkov ${ }^{123}$, A. Gomes ${ }^{139 a, 139 b}$, R. Goncalves Gama ${ }^{53}$, R. Gonçalo ${ }^{139 a, 139 c}$, G. Gonella ${ }^{131}$, L. Gonella ${ }^{21}$, A. Gongadze ${ }^{80}$, F. Gonnella ${ }^{21}$, J.L. Gonski ${ }^{39}$, S. González de la $\mathrm{Hoz}^{173}$, S. Gonzalez Fernandez ${ }^{14}$, R. Gonzalez Lopez ${ }^{91}$, C. Gonzalez Renteria ${ }^{18}$, R. Gonzalez Suarez ${ }^{171}$, S. Gonzalez-Sevilla ${ }^{54}$, G.R. Gonzalvo Rodriguez ${ }^{173}$, L. Goossens ${ }^{36}$, N.A. Gorasia ${ }^{21}$, P.A. Gorbounov ${ }^{124}$, H.A. Gordon ${ }^{29}$, B. Gorini ${ }^{36}$, E. Gorini ${ }^{68 a, 68 b}$, A. Gorišek ${ }^{92}$, A.T. Goshaw ${ }^{49}$, M.I. Gostkin ${ }^{80}$, C.A. Gottardo ${ }^{119}$, M. Gouighri ${ }^{35 b}$, A.G. Goussiou ${ }^{147}$, N. Govender ${ }^{33 c}$, C. Goy $^{5}$, I. Grabowska-Bold ${ }^{84 a}$, E.C. Graham ${ }^{91}$, J. Gramling ${ }^{170}$, E. Gramstad ${ }^{133}$, S. Grancagnolo ${ }^{19}$, M. Grandi ${ }^{155}$, V. Gratchev ${ }^{137}$, P.M. Gravila ${ }^{27 f}$, F.G. Gravili ${ }^{68 a}$,68b , C. Gray ${ }^{57}$, H.M. Gray ${ }^{18}$, C. Grefe ${ }^{24}$, K. Gregersen ${ }^{97}$, I.M. Gregor ${ }^{46}$, P. Grenier ${ }^{152}$, K. Grevtsov ${ }^{46}$, C. Grieco ${ }^{14}$, N.A. Grieser ${ }^{128}$, A.A. Grillo ${ }^{145}$, K. Grimm ${ }^{31, k}$, S. Grinstein ${ }^{14, w}$, J.-F. Grivaz ${ }^{65}$, S. Groh ${ }^{100}$, E. Gross ${ }^{179}$, J. Grosse-Knetter ${ }^{53}$, Z.J. Grout ${ }^{95}$, C. Grud ${ }^{106}$, A. Grummer ${ }^{118}$, J.C. Grundy ${ }^{134}$, L. Guan ${ }^{106}$, W. Guan ${ }^{180}$, C. Gubbels ${ }^{174}$, J. Guenther ${ }^{36}$, A. Guerguichon ${ }^{65}$, J.G.R. Guerrero Rojas ${ }^{173}$, F. Guescini ${ }^{115}$, D. Guest ${ }^{170}$, R. Gugel ${ }^{100}$, T. Guillemin ${ }^{5}$, S. Guindon ${ }^{36}$, U. Gul ${ }^{57}$,

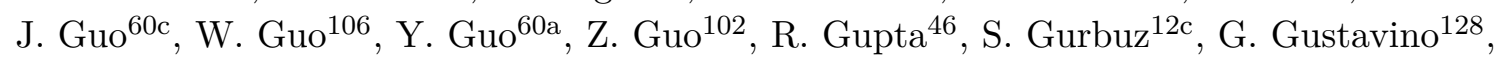
M. Guth ${ }^{52}$, P. Gutierrez ${ }^{128}$, C. Gutschow ${ }^{95}$, C. Guyot ${ }^{144}$, C. Gwenlan ${ }^{134}$,

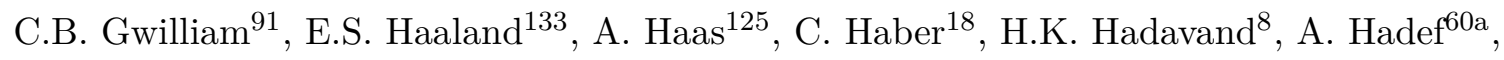
M. Haleem ${ }^{176}$, J. Haley ${ }^{129}$, J.J. Hall ${ }^{148}$, G. Halladjian ${ }^{107}$, G.D. Hallewell ${ }^{102}$,

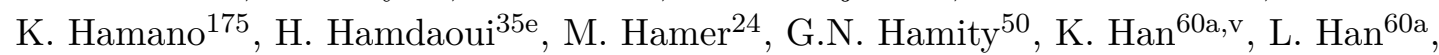
S. $\operatorname{Han}^{18}$, Y.F. $\operatorname{Han}^{166}$, K. Hanagaki ${ }^{82, t}$, M. Hance ${ }^{145}$, D.M. Handl ${ }^{114}$, M.D. Hank ${ }^{37}$, R. Hankache ${ }^{135}$, E. Hansen ${ }^{97}$, J.B. Hansen ${ }^{40}$, J.D. Hansen ${ }^{40}$, M.C. Hansen ${ }^{24}$, P.H. Hansen ${ }^{40}$, E.C. Hanson ${ }^{101}$, K. Hara ${ }^{168}$, T. Harenberg ${ }^{181}$, S. Harkusha ${ }^{108}$, P.F. Harrison ${ }^{177}$, N.M. Hartman ${ }^{152}$, N.M. Hartmann ${ }^{114}$, Y. Hasegawa ${ }^{149}$, A. Hasib ${ }^{50}$, S. Hassani ${ }^{144}$, S. $H_{a u g}^{20}$, R. Hauser ${ }^{107}$, L.B. Havener ${ }^{39}$, M. Havranek ${ }^{141}$, C.M. Hawkes ${ }^{21}$, R.J. Hawkings ${ }^{36}$, S. Hayashida ${ }^{117}$, D. Hayden ${ }^{107}$, C. Hayes ${ }^{106}$, R.L. Hayes ${ }^{174}$, C.P. Hays ${ }^{134}$, J.M. Hays ${ }^{93}$, H.S. Hayward ${ }^{91}$, S.J. Haywood ${ }^{143}$, F. He ${ }^{60 a}$, Y. $\mathrm{He}^{164}$, M.P. Heath ${ }^{50}$, V. Hedberg ${ }^{97}$, S. Heer ${ }^{24}$, A.L. Heggelund ${ }^{133}$, C. Heidegger ${ }^{52}$, K.K. Heidegger ${ }^{52}$, W.D. Heidorn ${ }^{79}$, J. Heilman ${ }^{34}$, S. Heim ${ }^{46}$, T. Heim ${ }^{18}$, B. Heinemann ${ }^{46, a j}$, J.G. Heinlein ${ }^{136}$, J.J. Heinrich ${ }^{131}$, L. Heinrich ${ }^{36}$, J. Hejbal ${ }^{140}$, L. Helary ${ }^{46}$, A. Held ${ }^{125}$, S. Hellesund ${ }^{133}$, C.M. Helling ${ }^{145}$, S. Hellman ${ }^{45 a, 45 b}$, C. Helsens ${ }^{36}$, R.C.W. Henderson ${ }^{90}$, Y. Heng ${ }^{180}$, L. Henkelmann ${ }^{32}$, A.M. Henriques Correia ${ }^{36}$, 
H. Herde ${ }^{26}$, Y. Hernández Jiménez ${ }^{33 e}$, H. Herr ${ }^{100}$, M.G. Herrmann ${ }^{114}$, T. Herrmann ${ }^{48}$, G. Herten ${ }^{52}$, R. Hertenberger ${ }^{114}$, L. Hervas ${ }^{36}$, T.C. Herwig ${ }^{136}$, G.G. Hesketh ${ }^{95}$, N.P. Hessey ${ }^{167 a}$, H. Hibi ${ }^{83}$, A. Higashida ${ }^{162}$, S. Higashino ${ }^{82}$, E. Higón-Rodriguez ${ }^{173}$, K. Hildebrand ${ }^{37}$, J.C. Hill ${ }^{32}$, K.K. Hill ${ }^{29}$, K.H. Hiller ${ }^{46}$, S.J. Hillier ${ }^{21}$, M. Hils ${ }^{48}$, I. Hinchliffe ${ }^{18}$, F. Hinterkeuser ${ }^{24}$, M. Hirose ${ }^{132}$, S. Hirose ${ }^{52}$, D. Hirschbuehl ${ }^{181}$, B. Hiti ${ }^{92}$, O. Hladik ${ }^{140}$, D.R. Hlaluku ${ }^{33 e}$, J. Hobbs ${ }^{154}$, N. Hod ${ }^{179}$, M.C. Hodgkinson ${ }^{148}$,

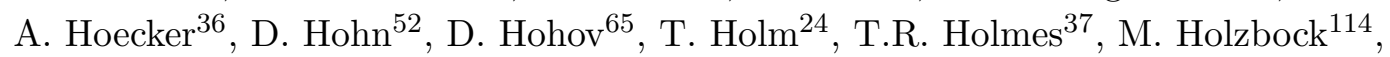
L.B.A.H. Hommels ${ }^{32}$, T.M. Hong ${ }^{138}$, J.C. Honig ${ }^{52}$, A. Hönle ${ }^{115}$, B.H. Hooberman ${ }^{172}$, W.H. Hopkins ${ }^{6}$, Y. Horii ${ }^{117}$, P. Horn ${ }^{48}$, L.A. Horyn ${ }^{37}$, S. Hou ${ }^{157}$, A. Hoummada ${ }^{35 a}$, J. Howarth ${ }^{57}$, J. Hoya ${ }^{89}$, M. Hrabovsky ${ }^{130}$, J. Hrdinka 77 , J. Hrivnac ${ }^{65}$, A. Hrynevich ${ }^{109}$, T. Hryn'ova ${ }^{5}$, P.J. Hsu ${ }^{64}$, S.-C. Hsu ${ }^{147}$, Q. $\mathrm{Hu}^{29}$, S. Hu${ }^{60 c}$, Y.F. Hu ${ }^{15 a, 15 d, a n}$, D.P. Huang ${ }^{95}$, Y. Huang ${ }^{60 a}$, Y. Huang ${ }^{15 a}$, Z. Hubacek ${ }^{141}$, F. Hubaut ${ }^{102}$, M. Huebner ${ }^{24}$, F. Huegging ${ }^{24}$, T.B. Huffman ${ }^{134}$, M. Huhtinen ${ }^{36}$, R. Hulsken ${ }^{58}$, R.F.H. Hunter ${ }^{34}$, P. Huo ${ }^{154}$, N. Huseynov ${ }^{80, a c}$, J. Huston ${ }^{107}$, J. Huth ${ }^{59}$, R. Hyneman ${ }^{106}$, S. Hyrych ${ }^{28 a}$, G. Iacobucci ${ }^{54}$, G. Iakovidis ${ }^{29}$, I. Ibragimov ${ }^{150}$, L. Iconomidou-Fayard ${ }^{65}$, P. Iengo ${ }^{36}$, R. Ignazzi ${ }^{40}$, O. Igonkina ${ }^{120, y, *}$, R. Iguchi ${ }^{162}$, T. Iizawa $^{54}$, Y. Ikegami ${ }^{82}$, M. Ikeno ${ }^{82}$,

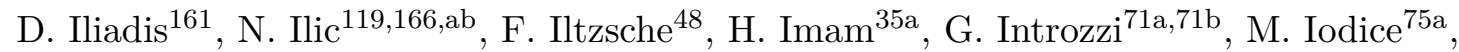

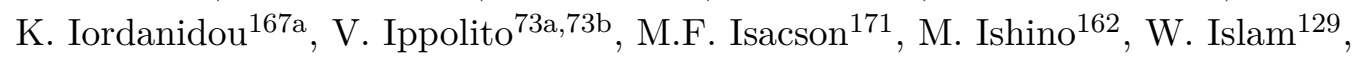
C. Issever ${ }^{19,46}$, S. Istin ${ }^{159}$, F. Ito ${ }^{168}$, J.M. Iturbe Ponce ${ }^{63 a}$, R. Iuppa ${ }^{76 a, 76 b}$, A. Ivina ${ }^{179}$, H. Iwasaki ${ }^{82}$, J.M. Izen ${ }^{43}$, V. Izzo ${ }^{70 a}$, P. Jacka ${ }^{140}$, P. Jackson ${ }^{1}$, R.M. Jacobs ${ }^{46}$, B.P. Jaeger ${ }^{151}$, V. Jain ${ }^{2}$, G. Jäkel ${ }^{181}$, K.B. Jakobi ${ }^{100}$, K. Jakobs ${ }^{52}$, T. Jakoubek ${ }^{179}$, J. Jamieson ${ }^{57}$, K.W. Janas ${ }^{84 a}$, R. Jansky ${ }^{54}$, M. Janus ${ }^{53}$, P.A. Janus ${ }^{84 a}$, G. Jarlskog ${ }^{97}$, A.E. Jaspan ${ }^{91}$, N. Javadov ${ }^{80, a c}$, T. Javưrek ${ }^{36}$, M. Javurkova ${ }^{103}$, F. Jeanneau ${ }^{144}$, L. Jeanty ${ }^{131}$, J. Jejelava ${ }^{158 a}$, P. Jenni ${ }^{52, c}$, N. Jeong ${ }^{46}$, S. Jézéquel ${ }^{5}$, H. Ji ${ }^{180}$, J. Jia ${ }^{154}$, H. Jiang ${ }^{79}$, Y. Jiang ${ }^{60 a}$, Z. Jiang ${ }^{152}$, S. Jiggins ${ }^{52}$, F.A. Jimenez Morales ${ }^{38}$, J. Jimenez Pena ${ }^{115}$, S. Jin ${ }^{15 c}$, A. Jinaru ${ }^{27 b}$, O. Jinnouchi ${ }^{164}$, H. Jivan ${ }^{33 e}$, P. Johansson ${ }^{148}$, K.A. Johns ${ }^{7}$, C.A. Johnson ${ }^{66}$, R.W.L. Jones ${ }^{90}$, S.D. Jones ${ }^{155}$, T.J. Jones ${ }^{91}$, J. Jongmanns ${ }^{61 a}$, J. Jovicevic ${ }^{36}$, X. Ju ${ }^{18}$, J.J. Junggeburth ${ }^{115}$, A. Juste Rozas ${ }^{14, w}$, A. Kaczmarska ${ }^{85}$, M. Kado ${ }^{73 a, 73 b}$, H. Kagan ${ }^{127}$, M. Kagan ${ }^{152}$, A. Kahn ${ }^{39}$, C. Kahra ${ }^{100}$, T. Kaji ${ }^{178}$, E. Kajomovitz ${ }^{159}$, C.W. Kalderon ${ }^{29}$, A. Kaluza ${ }^{100}$, A. Kamenshchikov ${ }^{123}$, M. Kaneda ${ }^{162}$, N.J. Kang ${ }^{145}$, S. Kang ${ }^{79}$, Y. Kano ${ }^{117}$, J. Kanzaki ${ }^{82}$, L.S. Kaplan ${ }^{180}$, D. Kar ${ }^{33 e}$, K. Karava ${ }^{134}$, M.J. Kareem ${ }^{167 b}$, I. Karkanias ${ }^{161}$, S.N. Karpov ${ }^{80}$, Z.M. Karpova ${ }^{80}$, V. Kartvelishvili ${ }^{90}$, A.N. Karyukhin ${ }^{123}$, A. Kastanas ${ }^{45 a, 45 b}$, C. Kato ${ }^{60 d, 60 c}$, J. Katzy ${ }^{46}$, K. Kawade ${ }^{149}$, K. Kawagoe ${ }^{88}$, T. Kawaguchi ${ }^{117}$, T. Kawamoto ${ }^{144}$, G. Kawamura ${ }^{53}$, E.F. Kay ${ }^{175}$, S. Kazakos ${ }^{14}$, V.F. Kazanin ${ }^{122 b, 122 a}$, R. Keeler ${ }^{175}$, R. Kehoe ${ }^{42}$, J.S. Keller ${ }^{34}$, E. Kellermann ${ }^{97}$, D. Kelsey ${ }^{155}$, J.J. Kempster ${ }^{21}$, J. Kendrick ${ }^{21}$, K.E. Kennedy ${ }^{39}$, O. Kepka ${ }^{140}$, S. Kersten ${ }^{181}$, B.P. Kerševan ${ }^{92}$, S. Ketabchi Haghighat ${ }^{166}$, M. Khader ${ }^{172}$, F. Khalil-Zada ${ }^{13}$, M. Khandoga ${ }^{144}$, A. Khanov ${ }^{129}$, A.G. Kharlamov ${ }^{122 b, 122 a}$, T. Kharlamova122b,122a, E.E. Khoda ${ }^{174}$, A. Khodinov ${ }^{165}$, T.J. Khoo ${ }^{54}$, G. Khoriauli ${ }^{176}$, E. Khramov ${ }^{80}$, J. Khubua ${ }^{158 b}$, S. Kido ${ }^{83}$, M. Kiehn ${ }^{54}$, C.R. Kilby ${ }^{94}$, E. Kim ${ }^{164}$, Y.K. Kim ${ }^{37}$, N. Kimura ${ }^{95}$, B.T. King ${ }^{91, *}$, A. Kirchhoff ${ }^{53}$, D. Kirchmeier ${ }^{48}$, J. Kirk ${ }^{143}$, A.E. Kiryunin ${ }^{115}$, T. Kishimoto ${ }^{162}$, D.P. Kisliuk ${ }^{166}$, V. Kitali ${ }^{46}$, C. Kitsaki ${ }^{10}$, O. Kivernyk ${ }^{24}$, T. Klapdor-Kleingrothaus ${ }^{52}$, 
M. Klassen ${ }^{61 a}$, C. Klein ${ }^{34}$, M.H. Klein ${ }^{106}$, M. Klein ${ }^{91}$, U. Klein ${ }^{91}$, K. Kleinknecht ${ }^{100}$, P. Klimek ${ }^{121}$, A. Klimentov ${ }^{29}$, T. Klingl ${ }^{24}$, T. Klioutchnikova ${ }^{36}$, F.F. Klitzner ${ }^{114}$, P. Kluit ${ }^{120}$, S. Kluth ${ }^{115}$, E. Kneringer ${ }^{77}$, E.B.F.G. Knoops ${ }^{102}$, A. Knue ${ }^{52}$, D. Kobayashi ${ }^{88}$, T. Kobayashi ${ }^{162}$, M. Kobel ${ }^{48}$, M. Kocian ${ }^{152}$, T. Kodama ${ }^{162}$, P. Kodys ${ }^{142}$, D.M. Koeck ${ }^{155}$, P.T. Koenig ${ }^{24}$, T. Koffas $^{34}$, N.M. Köhler ${ }^{36}$, M. Kolb ${ }^{144}$, I. Koletsou ${ }^{5}$, T. Komarek ${ }^{130}$, T. Kondo ${ }^{82}$, K. Köneke ${ }^{52}$, A.X.Y. Kong ${ }^{1}$, A.C. König ${ }^{119}$, T. Kono ${ }^{126}$,

V. Konstantinides ${ }^{95}$, N. Konstantinidis ${ }^{95}$, B. Konya ${ }^{97}$, R. Kopeliansky ${ }^{66}$, S. Koperny ${ }^{84 a}$, K. Korcyl ${ }^{85}$, K. Kordas ${ }^{161}$, G. Koren ${ }^{160}$, A. Korn ${ }^{95}$, I. Korolkov ${ }^{14}$, E.V. Korolkova ${ }^{148}$, N. Korotkova ${ }^{113}$, O. Kortner ${ }^{115}$, S. Kortner ${ }^{115}$, V.V. Kostyukhin ${ }^{148,165}$,

A. Kotsokechagia ${ }^{65}$, A. Kotwal ${ }^{49}$, A. Koulouris ${ }^{10}$, A. Kourkoumeli-Charalampidi ${ }^{71 a, 71 b}$, C. Kourkoumelis ${ }^{9}$, E. Kourlitis ${ }^{6}$, V. Kouskoura ${ }^{29}$, R. Kowalewski ${ }^{175}$, W. Kozanecki ${ }^{101}$, A.S. Kozhin ${ }^{123}$, V.A. Kramarenko ${ }^{113}$, G. Kramberger ${ }^{92}$, D. Krasnopevtsev ${ }^{60 a}$, M.W. Krasny ${ }^{135}$, A. Krasznahorkay ${ }^{36}$, D. Krauss ${ }^{115}$, J.A. Kremer ${ }^{100}$, J. Kretzschmar ${ }^{91}$, P. Krieger ${ }^{166}$, F. Krieter ${ }^{114}$, A. Krishnan ${ }^{61 b}$, K. Krizka ${ }^{18}$, K. Kroeninger ${ }^{47}$, H. Kroha ${ }^{115}$, J. Kroll ${ }^{140}$, J. Kroll ${ }^{136}$, K.S. Krowpman ${ }^{107}$, U. Kruchonak ${ }^{80}$, H. Krüger ${ }^{24}$,

N. Krumnack ${ }^{79}$, M.C. Kruse ${ }^{49}$, J.A. Krzysiak ${ }^{85}$, O. Kuchinskaia ${ }^{165}$, S. Kuday ${ }^{4 b}$, D. Kuechler ${ }^{46}$, J.T. Kuechler ${ }^{46}$, S. Kuehn ${ }^{36}$, T. Kuhl ${ }^{46}$, V. Kukhtin ${ }^{80}$, Y. Kulchitsky ${ }^{108, a e}$, S. Kuleshov ${ }^{146 b}$, Y.P. Kulinich ${ }^{172}$, M. Kuna ${ }^{58}$, T. Kunigo ${ }^{86}$, A. Kupco ${ }^{140}$, T. Kupfer ${ }^{47}$, O. Kuprash ${ }^{52}$, H. Kurashige ${ }^{83}$, L.L. Kurchaninov ${ }^{167 a}$, Y.A. Kurochkin ${ }^{108}$, A. Kurova ${ }^{112}$, M.G. Kurth ${ }^{15 a, 15 d}$, E.S. Kuwertz ${ }^{36}$, M. Kuze ${ }^{164}$, A.K. Kvam ${ }^{147}$, J. Kvita ${ }^{130}$, T. Kwan ${ }^{104}$, F. La Ruffa ${ }^{41 b, 41 a}$, C. Lacasta ${ }^{173}$, F. Lacava ${ }^{73 a, 73 b}$, D.P.J. Lack ${ }^{101}$, H. Lacker ${ }^{19}$, D. Lacour ${ }^{135}$, E. Ladygin ${ }^{80}$, R. Lafaye ${ }^{5}$, B. Laforge ${ }^{135}$, T. Lagouri ${ }^{146 b}$, S. Lai $^{53}$,

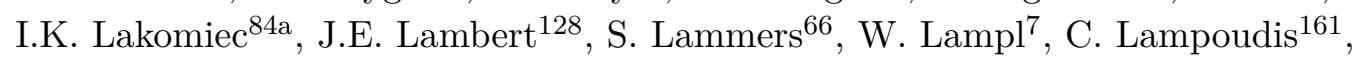
E. Lançon ${ }^{29}$, U. Landgraf ${ }^{52}$, M.P.J. Landon ${ }^{93}$, M.C. Lanfermann ${ }^{54}$, V.S. Lang ${ }^{52}$, J.C. Lange ${ }^{53}$, R.J. Langenberg ${ }^{103}$, A.J. Lankford ${ }^{170}$, F. Lanni ${ }^{29}$, K. Lantzsch ${ }^{24}$,

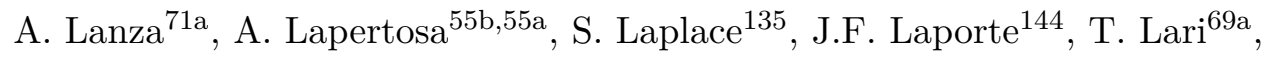
F. Lasagni Manghi23b,23a, M. Lassnig ${ }^{36}$, T.S. Lau ${ }^{63 a}$, A. Laudrain ${ }^{65}$, A. Laurier ${ }^{34}$, M. Lavorgna ${ }^{70 a, 70 b}$, S.D. Lawlor ${ }^{94}$, M. Lazzaroni ${ }^{69 a, 69 b}$, B. Le ${ }^{101}$, E. Le Guirriec $^{102}$, A. Lebedev ${ }^{79}$, M. LeBlanc ${ }^{7}$, T. LeCompte ${ }^{6}$, F. Ledroit-Guillon ${ }^{58}$, A.C.A. Lee ${ }^{95}$, C.A. Lee ${ }^{29}$, G.R. Lee ${ }^{17}$, L. Lee ${ }^{59}$, S.C. Lee ${ }^{157}$, S. Lee ${ }^{79}$, B. Lefebvre ${ }^{167 a}$, H.P. Lefebvre ${ }^{94}$, M. Lefebvre ${ }^{175}$, C. Leggett ${ }^{18}$, K. Lehmann ${ }^{151}$, N. Lehmann ${ }^{20}$, G. Lehmann Miotto ${ }^{36}$, W.A. Leight ${ }^{46}$, A. Leisos ${ }^{161, u}$, M.A.L. Leite ${ }^{81 d}$, C.E. Leitgeb ${ }^{114}$, R. Leitner ${ }^{142}$, D. Lellouch ${ }^{179,}{ }^{*}$, K.J.C. Leney ${ }^{42}$, T. Lenz ${ }^{24}$, S. Leone ${ }^{72 a}$, C. Leonidopoulos ${ }^{50}$, A. Leopold ${ }^{135}$, C. Leroy ${ }^{110}$, R. Les ${ }^{107}$, C.G. Lester ${ }^{32}$, M. Levchenko ${ }^{137}$, J. Levêque ${ }^{5}$, D. Levin ${ }^{106}$, L.J. Levinson ${ }^{179}$, D.J. Lewis ${ }^{21}$, B. Li ${ }^{15 b}$, B. Li ${ }^{106}$, C-Q. Li ${ }^{60 a}$, F. Li ${ }^{60 c}$,

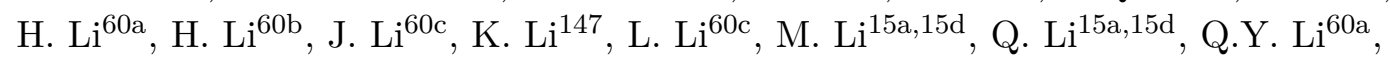

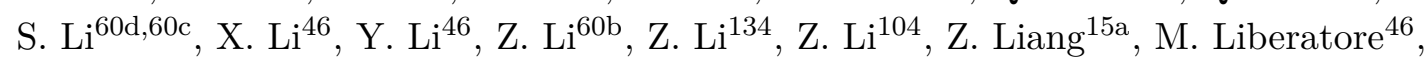
B. Liberti ${ }^{74 a}$, A. Liblong ${ }^{166}$, K. Lie ${ }^{63 c}$, S. $\operatorname{Lim}^{29}$, C.Y. $\operatorname{Lin}^{32}$, K. Lin ${ }^{107}$, R.A. Linck ${ }^{66}$, R.E. Lindley ${ }^{7}$, J.H. Lindon ${ }^{21}$, A. Linss ${ }^{46}$, A.L. Lionti ${ }^{54}$, E. Lipeles ${ }^{136}$, A. Lipniacka ${ }^{17}$, T.M. Liss ${ }^{172, a k}$, A. Lister ${ }^{174}$, J.D. Little ${ }^{8}$, B. Liu ${ }^{79}$, B.L. Liu ${ }^{6}$, H.B. Liu ${ }^{29}$, J.B. Liu ${ }^{60 a}$,

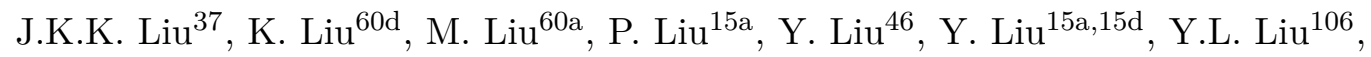

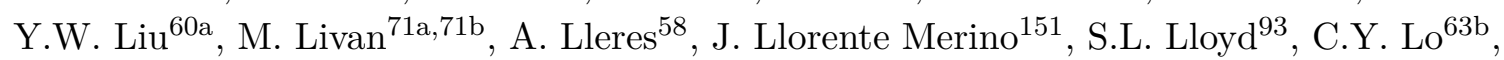
E.M. Lobodzinska ${ }^{46}$, P. Loch $^{7}$, S. Loffredo ${ }^{74 a, 74 b}$, T. Lohse ${ }^{19}$, K. Lohwasser ${ }^{148}$, 
M. Lokajicek ${ }^{140}$, J.D. Long ${ }^{172}$, R.E. Long ${ }^{90}$, I. Longarinii ${ }^{73 a, 73 b}$, L. Longo ${ }^{36}$, K.A. Looper ${ }^{127}$, I. Lopez Paz ${ }^{101}$, A. Lopez Solis ${ }^{148}$, J. Lorenz ${ }^{114}$, N. Lorenzo Martinez ${ }^{5}$,

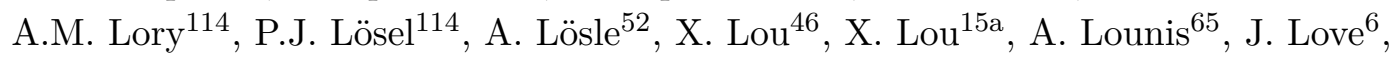
P.A. Love ${ }^{90}$, J.J. Lozano Bahilo ${ }^{173}$, M. Lu ${ }^{60 a}$, Y.J. Lu ${ }^{64}$, H.J. Lubatti ${ }^{147}$, C. Luci ${ }^{73 a, 73 b}$, F.L. Lucio Alves ${ }^{15 c}$, A. Lucotte $^{58}$, F. Luehring ${ }^{66}$, I. Luise ${ }^{135}$, L. Luminari ${ }^{73 a}$, B. Lund-Jensen ${ }^{153}$, M.S. Lutz ${ }^{160}$, D. Lynn ${ }^{29}$, H. Lyons ${ }^{91}$, R. Lysak ${ }^{140}$, E. Lytken ${ }^{97}$, F. Lyu ${ }^{15 a}$, V. Lyubushkin ${ }^{80}$, T. Lyubushkina ${ }^{80}$, H. Ma ${ }^{29}$, L.L. Ma ${ }^{60 b}$, Y. Ma ${ }^{95}$, D.M. Mac Donell ${ }^{175}$, G. Maccarrone ${ }^{51}$, A. Macchiolo ${ }^{115}$, C.M. Macdonald ${ }^{148}$, J.C. MacDonald ${ }^{148}$, J. Machado Miguens ${ }^{136}$, D. Madaffari ${ }^{173}$, R. Madar ${ }^{38}$, W.F. Mader ${ }^{48}$, M. Madugoda Ralalage Don ${ }^{129}$, N. Madysa ${ }^{48}$, J. Maeda ${ }^{83}$, T. Maeno ${ }^{29}$, M. Maerker ${ }^{48}$, V. Magerl ${ }^{52}$, N. Magini ${ }^{79}$, J. Magro67a,67c,q, D.J. Mahon $^{39}$, C. Maidantchik ${ }^{81 b}$, T. Maier ${ }^{114}$, A. Maio ${ }^{139 a, 139 b, 139 d}$, K. Maj ${ }^{84 a}$, O. Majersky ${ }^{28 a}$, S. Majewski ${ }^{131}$, Y. Makida ${ }^{82}$, N. Makovec ${ }^{65}$, B. Malaescu ${ }^{135}$, Pa. Malecki ${ }^{85}$, V.P. Maleev ${ }^{137}$, F. Malek ${ }^{58}$, D. Malito ${ }^{41 \mathrm{~b}, 41 \mathrm{a}}$, U. Mallik ${ }^{78}$, D. Malon ${ }^{6}$, C. Malone ${ }^{32}$, S. Maltezos ${ }^{10}$, S. Malyukov ${ }^{80}$, J. Mamuzic ${ }^{173}$, G. Mancini ${ }^{70 a, 70 b}$, I. Mandic ${ }^{92}$, L. Manhaes de Andrade Filho ${ }^{81 a}$, I.M. Maniatis ${ }^{161}$, J. Manjarres Ramos ${ }^{48}$, K.H. Mankinen ${ }^{97}$, A. Mann ${ }^{114}$, A. Manousos ${ }^{77}$, B. Mansoulie ${ }^{144}$, I. Manthos ${ }^{161}$, S. Manzoni ${ }^{120}$, A. Marantis ${ }^{161}$, G. Marceca ${ }^{30}$,

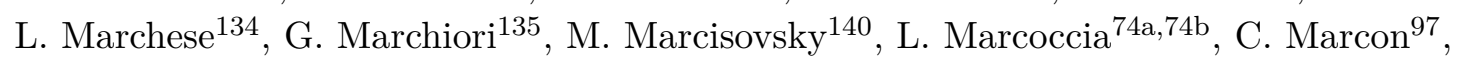
C.A. Marin Tobon ${ }^{36}$, M. Marjanovic ${ }^{128}$, Z. Marshall ${ }^{18}$, M.U.F. Martensson ${ }^{171}$,

S. Marti-Garcia ${ }^{173}$, C.B. Martin ${ }^{127}$, T.A. Martin ${ }^{177}$, V.J. Martin ${ }^{50}$,

B. Martin dit Latour ${ }^{17}$, L. Martinelli75a,75b, M. Martinez ${ }^{14, w}$, P. Martinez Agullo ${ }^{173}$, V.I. Martinez Outschoorn ${ }^{103}$, S. Martin-Haugh ${ }^{143}$, V.S. Martoiu ${ }^{27 b}$, A.C. Martyniuk ${ }^{95}$, A. Marzin $^{36}$, S.R. Maschek ${ }^{115}$, L. Masetti ${ }^{100}$, T. Mashimo ${ }^{162}$, R. Mashinistov ${ }^{111}$, J. Masik ${ }^{101}$, A.L. Maslennikov ${ }^{122 b, 122 a}$, L. Massa ${ }^{23 b, 23 a}$, P. Massarotti ${ }^{70 a, 70 b}$, P. Mastrandrea ${ }^{72 a, 72 b}$, A. Mastroberardino ${ }^{41 b, 41 a}$, T. Masubuchi ${ }^{162}$, D. Matakias ${ }^{29}$, A. Matic ${ }^{114}$, N. Matsuzawa ${ }^{162}$, P. Mättig ${ }^{24}$, J. Maurer ${ }^{27 b}$, B. Maček ${ }^{92}$, D.A. Maximov ${ }^{122 b, 122 a}$, R. Mazini ${ }^{157}$, I. Maznas ${ }^{161}$, S.M. Mazza ${ }^{145}$, J.P. Mc Gowan ${ }^{104}$, S.P. Mc Kee ${ }^{106}$, T.G. McCarthy ${ }^{115}$, W.P. McCormack ${ }^{18}$, E.F. McDonald ${ }^{105}$, J.A. Mcfayden ${ }^{36}$, G. Mchedlidze ${ }^{158 b}$, M.A. McKay ${ }^{42}$, K.D. McLean ${ }^{175}$, S.J. McMahon ${ }^{143}$, P.C. McNamara ${ }^{105}$, C.J. McNicol ${ }^{177}$, R.A. McPherson ${ }^{175, a b}$, J.E. Mdhluli ${ }^{33 e}$, Z.A. Meadows ${ }^{103}$, S. Meehan ${ }^{36}$, T. Megy ${ }^{38}$, S. Mehlhase ${ }^{114}$, A. Mehta ${ }^{91}$, B. Meirose ${ }^{43}$, D. Melini ${ }^{159}$, B.R. Mellado Garcia ${ }^{33 e}$, J.D. Mellenthin ${ }^{53}$, M. Melo ${ }^{28 a}$, F. Meloni ${ }^{46}$, A. Melzer ${ }^{24}$, E.D. Mendes Gouveia ${ }^{139 a, 139 e}$, L. Meng ${ }^{36}$, X.T. Meng ${ }^{106}$, S. Menke ${ }^{115}$, E. Meoni ${ }^{41 \mathrm{~b}, 41 \mathrm{a}}$, S. Mergelmeyer ${ }^{19}$, S.A.M. Merkt ${ }^{138}$, C. Merlassino ${ }^{134}$, P. Mermod $^{54}$, L. Merola ${ }^{70 a, 70 b}$, C. Meroni ${ }^{69 a}$, G. Merz ${ }^{106}$, O. Meshkov ${ }^{113,111}$, J.K.R. Meshreki ${ }^{150}$, J. Metcalfe $^{6}$, A.S. Mete ${ }^{6}$, C. Meyer ${ }^{66}$, J-P. Meyer ${ }^{144}$, M. Michetti ${ }^{19}$, R.P. Middleton ${ }^{143}$, L. Mijović ${ }^{50}$, G. Mikenberg ${ }^{179}$, M. Mikestikova ${ }^{140}$, M. Mikuž $^{92}$, H. Mildner ${ }^{148}$, A. Milic ${ }^{166}$, C.D. Milke ${ }^{42}$, D.W. Miller ${ }^{37}$, A. Milov $^{179}$, D.A. Milstead ${ }^{45 a, 45 b}$, R.A. Mina ${ }^{152}$, A.A. Minaenko ${ }^{123}$, I.A. Minashvilii ${ }^{158 b}$, A.I. Mincer ${ }^{125}$, B. Mindur ${ }^{84 a}$, M. Mineev ${ }^{80}$, Y. Minegishi ${ }^{162}$, L.M. Mir ${ }^{14}$, M. Mironova ${ }^{134}$, A. Mirto ${ }^{68 a, 68 b}$, K.P. Mistry ${ }^{136}$, T. Mitani ${ }^{178}$, J. Mitrevski ${ }^{114}$, V.A. Mitsou ${ }^{173}$, M. Mittal ${ }^{60 c}$, O. Miu ${ }^{166}$, A. Miucci ${ }^{20}$, P.S. Miyagawa ${ }^{93}$, A. Mizukami ${ }^{82}$, J.U. Mjörnmark ${ }^{97}$, T. Mkrtchyan ${ }^{61 a}$, M. Mlynarikova ${ }^{142}$, T. Moa ${ }^{45 a, 45 b}$, S. Mobius ${ }^{53}$, K. Mochizuki ${ }^{110}$, P. Mogg $^{114}$, 
S. Mohapatra ${ }^{39}$, R. Moles-Valls ${ }^{24}$, K. Mönig ${ }^{46}$, E. Monnier ${ }^{102}$, A. Montalbano ${ }^{151}$, J. Montejo Berlingen ${ }^{36}$, M. Montella ${ }^{95}$, F. Monticelli ${ }^{89}$, S. Monzani6 ${ }^{69 a}$, N. Morange ${ }^{65}$, D. Moreno ${ }^{22 a}$, M. Moreno Llácer ${ }^{173}$, C. Moreno Martinez ${ }^{14}$, P. Morettini ${ }^{55 b}$, M. Morgenstern ${ }^{159}$, S. Morgenstern ${ }^{48}$, D. Mori ${ }^{151}$, M. Morii ${ }^{59}$, M. Morinaga ${ }^{178}$, V. Morisbak ${ }^{133}$, A.K. Morley ${ }^{36}$, G. Mornacchi ${ }^{36}$, A.P. Morris ${ }^{95}$, L. Morvaj ${ }^{154}$, P. Moschovakos ${ }^{36}$, B. Moser ${ }^{120}$, M. Mosidze ${ }^{158 b}$, T. Moskalets ${ }^{144}$, H.J. Moss ${ }^{148}$, J. Moss ${ }^{31, m}$, E.J.W. Moyse ${ }^{103}$, S. Muanza ${ }^{102}$, J. Mueller ${ }^{138}$, R.S.P. Mueller ${ }^{114}$, D. Muenstermann ${ }^{90}$, G.A. Mullier ${ }^{97}$, D.P. Mungo ${ }^{69 a, 69 b}$, J.L. Munoz Martinez ${ }^{14}$, F.J. Munoz Sanchez ${ }^{101}$, P. Murin ${ }^{28 b}$, W.J. Murray ${ }^{177,143}$, A. Murrone ${ }^{69 a, 69 b}$, J.M. Muse ${ }^{128}$, M. Muškinja ${ }^{18}$, C. Mwewa ${ }^{33 a}$, A.G. Myagkov ${ }^{123, a g}$, A.A. Myers ${ }^{138}$, J. Myers ${ }^{131}$, M. Myska ${ }^{141}$, B.P. Nachman ${ }^{18}$, O. Nackenhorst ${ }^{47}$, A.Nag Nag ${ }^{48}$, K. Nagai $^{134}$, K. Nagano ${ }^{82}$, Y. Nagasaka ${ }^{62}$, J.L. Nagle ${ }^{29}$, E. Nagy ${ }^{102}$, A.M. Nairz ${ }^{36}$, Y. Nakahama ${ }^{117}$, K. Nakamura ${ }^{82}$, T. Nakamura ${ }^{162}$, H. Nanjo ${ }^{132}$, F. Napolitano ${ }^{61 a}$, R.F. Naranjo Garcia ${ }^{46}$, R. $\operatorname{Narayan}^{42}$, I. Naryshkin ${ }^{137}$, T. Naumann ${ }^{46}$, G. Navarro ${ }^{22 a}$, P.Y. Nechaeva ${ }^{111}$, F. Nechansky ${ }^{46}$, T.J. Neep ${ }^{21}$, A. Negri ${ }^{71 a}, 71 b$, M. Negrini ${ }^{23 b}$, C. Nellist ${ }^{119}$, C. Nelson ${ }^{104}$, M.E. Nelson ${ }^{45 a, 45 b}$, S. Nemecek ${ }^{140}$, M. Nessi ${ }^{36, e}$, M.S. Neubauer ${ }^{172}$, F. Neuhaus ${ }^{100}$, M. Neumann ${ }^{181}$, R. Newhouse ${ }^{174}$, P.R. Newman ${ }^{21}$, C.W. Ng ${ }^{138}$, Y.S. $\mathrm{Ng}^{19}$, Y.W.Y. $\mathrm{Ng}^{170}$, B. Ngair ${ }^{35 e}$, H.D.N. Nguyen ${ }^{102}$, T. Nguyen Manh ${ }^{110}$, E. Nibigira ${ }^{38}$, R.B. Nickerson ${ }^{134}$, R. Nicolaidou ${ }^{144}$, D.S. Nielsen ${ }^{40}$, J. Nielsen ${ }^{145}$, M. Niemeyer ${ }^{53}$, N. Nikiforou ${ }^{11}$, V. Nikolaenko ${ }^{123, a g}$, I. Nikolic-Audit ${ }^{135}$, K. Nikolopoulos ${ }^{21}$, P. Nilsson ${ }^{29}$, H.R. Nindhito ${ }^{54}$, Y. Ninomiya ${ }^{82}$, A. Nisati ${ }^{73 a}$, N. Nishu ${ }^{60 c}$, R. Nisius ${ }^{115}$, I. Nitsche ${ }^{47}$, T. Nitta ${ }^{178}$, T. Nobe ${ }^{162}$, D.L. Noel ${ }^{32}$, Y. Noguchi ${ }^{86}$, I. Nomidis ${ }^{135}$, M.A. Nomura ${ }^{29}$, M. Nordberg ${ }^{36}$, J. Novak ${ }^{92}$, T. Novak $^{92}$, O. Novgorodova ${ }^{48}$, R. Novotny ${ }^{141}$, L. Nozka ${ }^{130}$, K. Ntekas ${ }^{170}$, E. Nurse ${ }^{95}$, F.G. Oakham ${ }^{34, a l}$, H. Oberlack ${ }^{115}$, J. Ocariz ${ }^{135}$, A. Ochi ${ }^{83}$, I. Ochoa ${ }^{39}$, J.P. Ochoa-Ricoux ${ }^{146 a}$, K. O'Connor ${ }^{26}$, S. Oda $^{88}$, S. Odaka ${ }^{82}$, S. Oerdek $^{53}$, A. Ogrodnik ${ }^{84 a}$, A. $\mathrm{Oh}^{101}$, S.H. $\mathrm{Oh}^{49}$, C.C. $\mathrm{Ohm}^{153}$, H. Oide ${ }^{164}$, M.L. Ojeda ${ }^{166}$, H. Okawa ${ }^{168}$, Y. Okazaki ${ }^{86}$, M.W. O'Keefe ${ }^{91}$, Y. Okumura ${ }^{162}$, T. Okuyama ${ }^{82}$, A. Olariu ${ }^{27 b}$, L.F. Oleiro Seabra ${ }^{139 a}$, S.A. Olivares Pino ${ }^{146 a}$, D. Oliveira Damazio ${ }^{29}$, J.L. Oliver ${ }^{1}$, M.J.R. Olsson ${ }^{170}$, A. Olszewski ${ }^{85}$, J. Olszowska ${ }^{85}$, Ö.O. Öncel ${ }^{24}$, D.C. O'Neil ${ }^{151}$, A.P. O'neill ${ }^{134}$, A. Onofre ${ }^{139 a, 139 e}$, P.U.E. Onyisi ${ }^{11}$, H. Oppen ${ }^{133}$, R.G. Oreamuno Madriz ${ }^{121}$, M.J. Oreglia ${ }^{37}$, G.E. Orellana ${ }^{89}$, D. Orestano ${ }^{75 a, 75 b}$, N. Orlando ${ }^{14}$, R.S. Orr ${ }^{166}$, V. O'Shea ${ }^{57}$, R. Ospanov ${ }^{60 a}$, G. Otero y Garzon ${ }^{30}$, H. Otono ${ }^{88}$, P.S. Ott ${ }^{61 a}$, G.J. Ottino ${ }^{18}$, M. Ouchrif ${ }^{35 d}$, J. Ouellette ${ }^{29}$, F. Ould-Saada ${ }^{133}$, A. Ouraou ${ }^{144}$, Q. Ouyang ${ }^{15 a}$, M. Owen ${ }^{57}$, R.E. Owen ${ }^{143}$, V.E. Ozcan ${ }^{12 c}$, N. Ozturk ${ }^{8}$, J. Pacalt ${ }^{130}$, H.A. Pacey ${ }^{32}$, K. Pachal ${ }^{49}$, A. Pacheco Pages ${ }^{14}$, C. Padilla Aranda ${ }^{14}$, S. Pagan Griso ${ }^{18}$, G. Palacino ${ }^{66}$, S. Palazzo ${ }^{50}$, S. Palestini ${ }^{36}$, M. Palka ${ }^{84 b}$, P. Palni ${ }^{84 a}$, C.E. Pandini ${ }^{54}$, J.G. Panduro Vazquez ${ }^{94}$, P. Pani ${ }^{46}$, G. Panizzo ${ }^{67 a, 67 c}$, L. Paolozzi ${ }^{54}$, C. Papadatos ${ }^{110}$, K. Papageorgiou ${ }^{9, g}$, S. Parajuli ${ }^{42}$, A. Paramonov ${ }^{6}$, C. Paraskevopoulos ${ }^{10}$, D. Paredes Hernandez ${ }^{63 b}$, S.R. Paredes Saenz ${ }^{134}$, B. Parida ${ }^{179}$, T.H. Park ${ }^{166}$, A.J. Parker ${ }^{31}$, M.A. Parker ${ }^{32}$, F. Parodi ${ }^{55 b, 55 a, ~ E . W . ~ P a r r i s h ~}{ }^{121}$, J.A. Parsons ${ }^{39}$, U. Parzefall ${ }^{52}$, L. Pascual Dominguez ${ }^{135}$, V.R. Pascuzzi ${ }^{18}$, J.M.P. Pasner ${ }^{145}$, F. Pasquali ${ }^{120}$, E. Pasqualucci ${ }^{73 a}$, S. Passaggio ${ }^{55 b}$, F. Pastore ${ }^{94}$, P. Pasuwan ${ }^{45 a, 45 b}$, S. Pataraia ${ }^{100}$, J.R. Pater ${ }^{101}$, A. Pathak ${ }^{180, i}$, J. Patton ${ }^{91}$, T. Pauly ${ }^{36}$, 
J. Pearkes ${ }^{152}$, B. Pearson ${ }^{115}$, M. Pedersen ${ }^{133}$, L. Pedraza Diaz ${ }^{119}$, R. Pedro ${ }^{139 a}$, T. Peiffer ${ }^{53}$, S.V. Peleganchuk ${ }^{122 b, 122 a}$, O. Penc ${ }^{140}$, H. Peng ${ }^{60 a}$, B.S. Peralva ${ }^{81 a}$, M.M. Perego ${ }^{65}$, A.P. Pereira Peixoto ${ }^{139 a}$, L. Pereira Sanchez ${ }^{45 a, 45 b}$, D.V. Perepelitsa ${ }^{29}$, E. Perez Codina ${ }^{167 a}$, F. Peri ${ }^{19}$, L. Perini ${ }^{69 a, 69 b}$, H. Pernegger ${ }^{36}$, S. Perrella ${ }^{36}$, A. Perrevoort ${ }^{120}$, K. Peters ${ }^{46}$, R.F.Y. Peters ${ }^{101}$, B.A. Petersen ${ }^{36}$, T.C. Petersen ${ }^{40}$, E. Petit ${ }^{102}$, V. Petousis ${ }^{141}$, A. Petridis ${ }^{1}$, C. Petridou ${ }^{161}$, F. Petrucci ${ }^{75 a, 75 b}$, M. Pettee $^{182}$, N.E. Pettersson ${ }^{103}$, K. Petukhova ${ }^{142}$, A. Peyaud ${ }^{144}$, R. Pezoa ${ }^{146 d}$, L. Pezzotti ${ }^{71 a, 71 b}$, T. Pham ${ }^{105}$, F.H. Phillips ${ }^{107}$, P.W. Phillips ${ }^{143}$, M.W. Phipps ${ }^{172}$, G. Piacquadio ${ }^{154}$, E. Pianori ${ }^{18}$, A. Picazio ${ }^{103}$, R.H. Pickles ${ }^{101}$, R. Piegaia ${ }^{30}$, D. Pietreanu ${ }^{27 b}$, J.E. Pilcher ${ }^{37}$, A.D. Pilkington ${ }^{101}$, M. Pinamonti ${ }^{67 a, 67 c}$, J.L. Pinfold ${ }^{3}$, C. Pitman Donaldson ${ }^{95}$, M. Pitt ${ }^{160}$, L. Pizzimento ${ }^{74 a, 74 b}$, M.-A. Pleier ${ }^{29}$, V. Pleskot ${ }^{142}$, E. Plotnikova ${ }^{80}$, P. Podberezko ${ }^{122 b, 122 a}$, R. Poettgen ${ }^{97}$, R. Poggi ${ }^{54}$, L. Poggioli ${ }^{135}$, I. Pogrebnyak ${ }^{107}$, D. Pohl ${ }^{24}$, I. Pokharel ${ }^{53}$, G. Polesello ${ }^{71 a}$, A. Poley ${ }^{151,167 a}$, A. Policicchio ${ }^{73 a, 73 b}$, R. Polifka ${ }^{142}$, A. Polini ${ }^{23 b}$, C.S. Pollard ${ }^{46}$, V. Polychronakos ${ }^{29}$, D. Ponomarenko ${ }^{112}$, L. Pontecorvo ${ }^{36}$, S. Popa ${ }^{27 a}$, G.A. Popeneciu ${ }^{27 d}$, L. Portales ${ }^{5}$, D.M. Portillo Quintero ${ }^{58}$, S. Pospisil ${ }^{141}$, K. Potamianos ${ }^{46}$, I.N. Potrap ${ }^{80}$, C.J. Potter ${ }^{32}$, H. Potti ${ }^{11}$, T. Poulsen ${ }^{97}$, J. Poveda ${ }^{173}$, T.D. Powell ${ }^{148}$, G. Pownall ${ }^{46}$, M.E. Pozo Astigarraga ${ }^{36}$, P. Pralavorio ${ }^{102}$, S. Prell ${ }^{79}$, D. Price ${ }^{101}$, M. Primavera ${ }^{68 a}$, M.L. Proffitt ${ }^{147}$, N. Proklova ${ }^{112}$, K. Prokofiev ${ }^{63 c}$, F. Prokoshin ${ }^{80}$, S. Protopopescu ${ }^{29}$, J. Proudfoot ${ }^{6}$, M. Przybycien ${ }^{84 a}$, D. Pudzha ${ }^{137}$, A. Puri ${ }^{172}$, P. Puzo ${ }^{65}$, D. Pyatiizbyantseva ${ }^{112}$, J. Qian ${ }^{106}$, Y. Qin ${ }^{101}$, A. Quadt ${ }^{53}$,

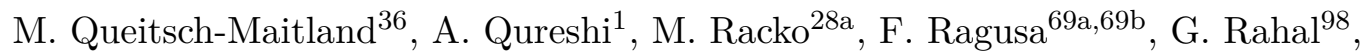
J.A. Raine ${ }^{54}$, S. Rajagopalan ${ }^{29}$, A. Ramirez Morales ${ }^{93}$, K. Ran ${ }^{15 a, 15 d}$, D.M. Rauch ${ }^{46}$, F. Rauscher ${ }^{114}$, S. Rave $^{100}$, B. Ravina ${ }^{148}$, I. Ravinovich ${ }^{179}$, J.H. Rawling ${ }^{101}$, M. Raymond ${ }^{36}$, A.L. $\operatorname{Read}^{133}$, N.P. Readioff ${ }^{58}$, M. Reale ${ }^{68 a, 68 b}$, D.M. Rebuzzi ${ }^{71 a, 71 b}$, G. Redlinger ${ }^{29}$, K. Reeves ${ }^{43}$, J. Reichert ${ }^{136}$, D. Reikher ${ }^{160}$, A. Reiss ${ }^{100}$, A. Rej $^{150}$, C. Rembser ${ }^{36}$, A. Renardi ${ }^{46}$, M. Renda ${ }^{27 b}$, M.B. Rendel ${ }^{115}$, S. Resconi ${ }^{69 a}$, E.D. Resseguie ${ }^{18}$, S. Rettie $^{95}$, B. Reynolds ${ }^{127}$, E. Reynolds ${ }^{21}$, O.L. Rezanova ${ }^{122 b, 122 a}$, P. Reznicek ${ }^{142}$, E. Ricci ${ }^{76 a, 76 b}$, R. Richter ${ }^{115}$, S. Richter ${ }^{46}$, E. Richter-Was ${ }^{84 b}$, M. Ridel ${ }^{135}$, P. Rieck ${ }^{115}$, O. Rifki ${ }^{46}$, M. Rijssenbeek ${ }^{154}$, A. Rimoldi ${ }^{71 a, 71 b}$, M. Rimoldi ${ }^{46}$, L. Rinaldi ${ }^{23 b}$, T.T. Rinn ${ }^{172}$, G. Ripellino ${ }^{153}$, I. Riu ${ }^{14}$, P. Rivadeneira ${ }^{46}$, J.C. Rivera Vergara ${ }^{175}$, F. Rizatdinova ${ }^{129}$, E. Rizvi ${ }^{93}$, C. Rizzi ${ }^{36}$, S.H. Robertson ${ }^{104, a b}$, M. Robin ${ }^{46}$, D. Robinson ${ }^{32}$, C.M. Robles Gajardo ${ }^{146 \mathrm{~d}}$, M. Robles Manzano ${ }^{100}$, A. Robson ${ }^{57}$, A. Rocchi ${ }^{74 a, 74 b}$, E. Rocco ${ }^{100}$, C. Roda ${ }^{72 a, 72 b}$, S. Rodriguez Bosca ${ }^{173}$, A.M. Rodríguez Vera ${ }^{167 b}$, S. Roe ${ }^{36}$, J. Roggel ${ }^{181}$, O. Røhne ${ }^{133}$, R. Röhrig ${ }^{115}$, R.A. Rojas ${ }^{146 \mathrm{~d}}$, B. Roland ${ }^{52}$, C.P.A. Roland ${ }^{66}$, J. Roloff ${ }^{29}$, A. Romaniouk ${ }^{112}$, M. Romano ${ }^{23 b, 23 a}$, N. Rompotis ${ }^{91}$, M. Ronzani ${ }^{125}$, L. Roos ${ }^{135}$, S. Rosati ${ }^{73 a}$, G. Rosin ${ }^{103}$, B.J. Rosser ${ }^{136}$, E. Rossi ${ }^{46}$, E. Rossi ${ }^{75 a, 75 b}$, E. Rossi ${ }^{70 a, 70 b}$, L.P. Rossi ${ }^{55 b}$, L. Rossini ${ }^{69 a, 69 b}$,

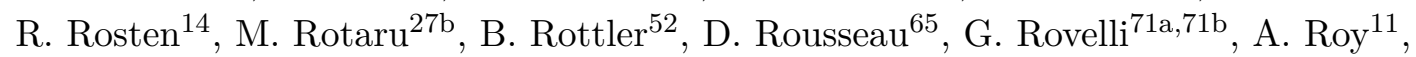
D. Roy ${ }^{33 e}$, A. Rozanov ${ }^{102}$, Y. Rozen ${ }^{159}$, X. Ruan ${ }^{33 e}$, F. Rühr ${ }^{52}$, A. Ruiz-Martinez ${ }^{173}$, A. Rummler ${ }^{36}$, Z. Rurikova ${ }^{52}$, N.A. Rusakovich ${ }^{80}$, H.L. Russell ${ }^{104}$, L. Rustige ${ }^{38,47}$, J.P. Rutherfoord 7 , E.M. Rüttinger ${ }^{148}$, M. Rybar $^{39}$, G. Rybkin ${ }^{65}$, E.B. Rye ${ }^{133}$, A. Ryzhov ${ }^{123}$, J.A. Sabater Iglesias ${ }^{46}$, P. Sabatini ${ }^{53}$, L. Sabetta ${ }^{73 a, 73 b}$, S. Sacerdoti ${ }^{65}$, H.F-W. Sadrozinski ${ }^{145}$, R. Sadykov ${ }^{80}$, F. Safai Tehrani ${ }^{73 a}$, B. Safarzadeh Samani ${ }^{155}$, 
M. Safdari ${ }^{152}$, P. Saha ${ }^{121}$, S. Saha ${ }^{104}$, M. Sahinsoy ${ }^{115}$, A. Sahu ${ }^{181}$, M. Saimpert ${ }^{36}$, M. Saito ${ }^{162}$, T. Saito ${ }^{162}$, H. Sakamoto ${ }^{162}$, D. Salamani ${ }^{54}$, G. Salamanna ${ }^{75 a, 75 b}$, A. Salnikov ${ }^{152}$, J. Salt ${ }^{173}$, A. Salvador Salas ${ }^{14}$, D. Salvatore ${ }^{41 b, 41 a}$, F. Salvatore ${ }^{155}$, A. Salvucci ${ }^{63 a, 63 b, 63 c}$, A. Salzburger ${ }^{36}$, J. Samarati ${ }^{36}$, D. Sammel ${ }^{52}$, D. Sampsonidis ${ }^{161}$, D. Sampsonidou ${ }^{161}$, J. Sánchez ${ }^{173}$, A. Sanchez Pineda ${ }^{67 a, 36,67 c}$, H. Sandaker ${ }^{133}$, C.O. Sander ${ }^{46}$, I.G. Sanderswood ${ }^{90}$, M. Sandhoff ${ }^{181}$, C. Sandoval ${ }^{22 a}$, D.P.C. Sankey ${ }^{143}$, M. Sannino ${ }^{55 b, 55 a}$, Y. Sano ${ }^{117}$, A. Sansoni ${ }^{51}$, C. Santoni ${ }^{38}$, H. Santos ${ }^{139 a, 139 b}$, S.N. Santpur ${ }^{18}$, A. Santra ${ }^{173}$, K.A. Saoucha ${ }^{148}$, A. Sapronov ${ }^{80}$, J.G. Saraiva ${ }^{139 a, 139 d}$, O. Sasaki ${ }^{82}$, K. Sato ${ }^{168}$, F. Sauerburger ${ }^{52}$, E. Sauvan ${ }^{5}$, P. Savard ${ }^{166, a l}$, R. Sawada ${ }^{162}$, C. Sawyer ${ }^{143}$, L. Sawyer ${ }^{96, a f}$, I. Sayago Galvan ${ }^{173}$, C. Sbarra ${ }^{23 b}$, A. Sbrizzi ${ }^{67 a, 67 c}$, T. Scanlon ${ }^{95}$, J. Schaarschmidt ${ }^{147}$, P. Schacht ${ }^{115}$, D. Schaefer ${ }^{37}$, L. Schaefer ${ }^{136}$, S. Schaepe ${ }^{36}$, U. Schäfer ${ }^{100}$, A.C. Schaffer ${ }^{65}$, D. Schaile ${ }^{114}$, R.D. Schamberger ${ }^{154}$, E. Schanet ${ }^{114}$, N. Scharmberg ${ }^{101}$, V.A. Schegelsky ${ }^{137}$, D. Scheirich ${ }^{142}$, F. Schenck ${ }^{19}$, M. Schernau ${ }^{170}$, C. Schiavi ${ }^{55 b, 55 a}$, L.K. Schildgen ${ }^{24}$, Z.M. Schillaci ${ }^{26}$, E.J. Schioppa ${ }^{68 a, 68 b}$, M. Schioppa ${ }^{41 b, 41 a}$, K.E. Schleicher ${ }^{52}$, S. Schlenker ${ }^{36}$, K.R. Schmidt-Sommerfeld ${ }^{115}$, K. Schmieden ${ }^{36}$, C. Schmitt ${ }^{100}$, S. Schmitt ${ }^{46}$, J.C. Schmoeckel ${ }^{46}$, L. Schoeffel ${ }^{144}$, A. Schoening ${ }^{61 b}$, P.G. Scholer ${ }^{52}$, E. Schopf ${ }^{134}$, M. Schott ${ }^{100}$, J.F.P. Schouwenberg ${ }^{119}$, J. Schovancova ${ }^{36}$, S. Schramm ${ }^{54}$, F. Schroeder ${ }^{181}$, A. Schulte ${ }^{100}$, H-C. Schultz-Coulon ${ }^{61 a}$, M. Schumacher ${ }^{52}$, B.A. Schumm ${ }^{145}$, Ph. Schune ${ }^{144}$, A. Schwartzman ${ }^{152}$, T.A. Schwarz ${ }^{106}$, Ph. Schwemling ${ }^{144}$, R. Schwienhorst ${ }^{107}$, A. Sciandra ${ }^{145}$, G. Sciolla ${ }^{26}$,

M. Scornajenghi ${ }^{41 b, 41 a}$, F. Scuri ${ }^{72 a}$, F. Scutti ${ }^{105}$, L.M. Scyboz ${ }^{115}$, C.D. Sebastiani ${ }^{91}$, P. Seema ${ }^{19}$, S.C. Seidel ${ }^{118}$, A. Seiden ${ }^{145}$, B.D. Seidlitz ${ }^{29}$, T. Seiss ${ }^{37}$, C. Seitz ${ }^{46}$, J.M. Seixas ${ }^{81 b}$, G. Sekhniaidze ${ }^{70 a}$, S.J. Sekula ${ }^{42}$, N. Semprini-Cesari ${ }^{23 b, 23 a}{ }^{2}$ S. Sen ${ }^{49}$, C. Serfon ${ }^{29}$, L. Serin ${ }^{65}$, L. Serkin ${ }^{67 a, 67 b}$, M. Sessa ${ }^{60 a}$, H. Severini ${ }^{128}$, S. Sevova ${ }^{152}$, F. Sforza ${ }^{55 b, 55 a}$, A. Sfyrla ${ }^{54}$, E. Shabalina ${ }^{53}$, J.D. Shahinian ${ }^{145}$, N.W. Shaikh ${ }^{45 a, 45 b}$, D. Shaked Renous ${ }^{179}$, L.Y. Shan ${ }^{15 a}$, M. Shapiro ${ }^{18}$, A. Sharma ${ }^{134}$, A.S. Sharma ${ }^{1}$, P.B. Shatalov ${ }^{124}$, K. Shaw ${ }^{155}$, S.M. Shaw ${ }^{101}$, M. Shehade ${ }^{179}$, Y. Shen ${ }^{128}$, A.D. Sherman ${ }^{25}$, P. Sherwood ${ }^{95}$, L. Shi ${ }^{95}$, S. Shimizu ${ }^{82}$, C.O. Shimmin ${ }^{182}$, Y. Shimogama ${ }^{178}$, M. Shimojima ${ }^{116}$, I.P.J. Shipsey ${ }^{134}$, S. Shirabe ${ }^{164}$, M. Shiyakova ${ }^{80, z}$, J. Shlomi ${ }^{179}$, A. Shmeleva ${ }^{111}$, M.J. Shochet ${ }^{37}$, J. Shojaii ${ }^{105}$, D.R. Shope ${ }^{128}$, S. Shrestha ${ }^{127}$, E.M. Shrif ${ }^{33 e}$, E. Shulga ${ }^{179}$, P. Sicho ${ }^{140}$, A.M. Sickles ${ }^{172}$, E. Sideras Haddad ${ }^{33 e}$, O. Sidiropoulou ${ }^{36}$, A. Sidoti ${ }^{23 b, 23 a}$, F. Siegert ${ }^{48}$, Dj. Sijacki ${ }^{16}$, M.Jr. Silva ${ }^{180}$, M.V. Silva Oliveira ${ }^{36}$, S.B. Silverstein ${ }^{45 a}$, S. Simion ${ }^{65}$, R. Simoniello ${ }^{100}$, C.J. Simpson-allsop ${ }^{21}$, S. Simsek ${ }^{12 b}$, P. Sinervo ${ }^{166}$, V. Sinetckii ${ }^{113}$, S. Singh ${ }^{151}$, M. Sioli ${ }^{23 b, 23 a}$, I. Siral ${ }^{131}$, S.Yu. Sivoklokov ${ }^{113}$, J. Sjölin ${ }^{45 a, 45 b}$, A. Skaf ${ }^{53}$, E. Skorda ${ }^{97}$, P. Skubic ${ }^{128}$, M. Slawinska ${ }^{85}$, K. Sliwa ${ }^{169}$, R. Slovak ${ }^{142}$, V. Smakhtin ${ }^{179}$, B.H. Smart ${ }^{143}$, J. Smiesko ${ }^{28 b}$, N. Smirnov ${ }^{112}$, S.Yu. Smirnov ${ }^{112}$, Y. Smirnov ${ }^{112}$, L.N. Smirnova ${ }^{113, r}$, O. Smirnova ${ }^{97}$, H.A. Smith ${ }^{134}$, M. Smizanska ${ }^{90}$, K. Smolek ${ }^{141}$, A. Smykiewicz ${ }^{85}$, A.A. Snesarev ${ }^{111}$, H.L. Snoek ${ }^{120}$, I.M. Snyder ${ }^{131}$, S. Snyder ${ }^{29}$, R. Sobie ${ }^{175, a b}$, A. Soffer ${ }^{160}$, A. Søgaard ${ }^{50}$, F. Sohns ${ }^{53}$, C.A. Solans Sanchez ${ }^{36}$, E.Yu. Soldatov ${ }^{112}$, U. Soldevila ${ }^{173}$, A.A. Solodkov ${ }^{123}$, A. Soloshenko ${ }^{80}$, O.V. Solovyanov ${ }^{123}$, V. Solovyev ${ }^{137}$, P. Sommer ${ }^{148}$, H. Son ${ }^{169}$, W. Song ${ }^{143}$, W.Y. Song ${ }^{167 b}$, A. Sopczak ${ }^{141}$, A.L. Sopio ${ }^{95}$, F. Sopkova ${ }^{28 b}$, S. Sottocornola ${ }^{71 a, 71 b}$, R. Soualah ${ }^{67 a, 67 c}$, A.M. Soukharev ${ }^{122 b, 122 a}$, D. South ${ }^{46}$, 
S. Spagnolo ${ }^{68 a, 68 b}$, M. Spalla ${ }^{115}$, M. Spangenberg ${ }^{177}$, F. Spanò ${ }^{94}$, D. Sperlich ${ }^{52}$, T.M. Spieker ${ }^{61 a}$, G. Spigo ${ }^{36}$, M. Spina ${ }^{155}$, D.P. Spiteri ${ }^{57}$, M. Spousta ${ }^{142}$, A. Stabile ${ }^{69 a, 69 b}$, B.L. Stamas ${ }^{121}$, R. Stamen ${ }^{61 a}$, M. Stamenkovic ${ }^{120}$, E. Stanecka ${ }^{85}$, B. Stanislaus ${ }^{134}$, M.M. Stanitzki ${ }^{46}$, M. Stankaityte ${ }^{134}$, B. Stapf ${ }^{120}$, E.A. Starchenko ${ }^{123}$, G.H. Stark ${ }^{145}$, J. Stark ${ }^{58}$, P. Staroba ${ }^{140}$, P. Starovoitov ${ }^{61 a}$, S. Stärz ${ }^{104}$, R. Staszewski ${ }^{85}$, G. Stavropoulos ${ }^{44}$, M. Stegler ${ }^{46}$, P. Steinberg ${ }^{29}$, A.L. Steinhebel ${ }^{131}$, B. Stelzer ${ }^{151}$, H.J. Stelzer ${ }^{138}$, O. Stelzer-Chilton ${ }^{167 a}$, H. Stenzel ${ }^{56}$, T.J. Stevenson ${ }^{155}$, G.A. Stewart ${ }^{36}$, M.C. Stockton ${ }^{36}$, G. Stoicea ${ }^{27 b}$, M. Stolarski ${ }^{139 a}$, S. Stonjek ${ }^{115}$, A. Straessner ${ }^{48}$, J. Strandberg ${ }^{153}$, S. Strandberg ${ }^{45 a, 45 b}$, M. Strauss ${ }^{128}$, T. Strebler ${ }^{102}$, P. Strizenec ${ }^{28 b}$, R. Ströhmer ${ }^{176}$, D.M. Strom ${ }^{131}$, R. Stroynowski ${ }^{42}$, A. Strubig ${ }^{50}$, S.A. Stucci ${ }^{29}$, B. Stugu ${ }^{17}$, J. Stupak ${ }^{128}$, N.A. Styles ${ }^{46}$, D. Su ${ }^{152}$, W. Su ${ }^{60 c, 147}$, S. Suchek ${ }^{61 a}$, V.V. Sulin ${ }^{111}$, M.J. Sullivan ${ }^{91}$, D.M.S. Sultan ${ }^{54}$, S. Sultansoy ${ }^{4 c}$, T. Sumida ${ }^{86}$, S. Sun ${ }^{106}$, X. Sun ${ }^{101}$, K. Suruliz ${ }^{155}$, C.J.E. Suster ${ }^{156}$, M.R. Sutton ${ }^{155}$, S. Suzuki ${ }^{82}$, M. Svatos ${ }^{140}$, M. Swiatlowski ${ }^{167 a}$, S.P. Swift ${ }^{2}$, T. Swirski ${ }^{176}$, A. Sydorenko ${ }^{100}$, I. Sykora ${ }^{28 a}$,

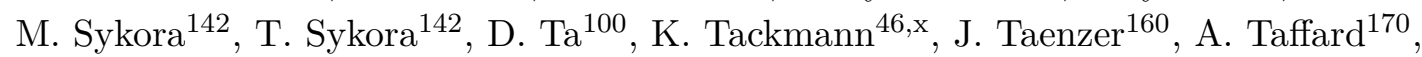
R. Tafirout ${ }^{167 a}$, R. Takashima ${ }^{87}$, K. Takeda ${ }^{83}$, T. Takeshita ${ }^{149}$, E.P. Takeva ${ }^{50}$, Y. Takubo ${ }^{82}$, M. Talby ${ }^{102}$, A.A. Talyshev ${ }^{122 b, 122 a}$, K.C. Tam ${ }^{63 b}$, N.M. Tamir ${ }^{160}$, J. Tanaka ${ }^{162}$, R. Tanaka ${ }^{65}$, S. Tapia Araya ${ }^{172}$, S. Tapprogge ${ }^{100}$,

A. Tarek Abouelfadl Mohamed ${ }^{107}$, S. Tarem ${ }^{159}$, K. Tariq ${ }^{60 b}$, G. Tarna ${ }^{27 b, d}$, G.F. Tartarelli ${ }^{69 a}$, P. Tas ${ }^{142}$, M. Tasevsky ${ }^{140}$, T. Tashiro ${ }^{86}$, E. Tassi ${ }^{41 b, 41 a}$,

A. Tavares Delgado ${ }^{139 a}$, Y. Tayalati ${ }^{35 e}$, A.J. Taylor ${ }^{50}$, G.N. Taylor ${ }^{105}$, W. Taylor ${ }^{167 b}$, H. Teagle ${ }^{91}$, A.S. Tee ${ }^{90}$, R. Teixeira De Lima ${ }^{152}$, P. Teixeira-Dias ${ }^{94}$, H. Ten Kate ${ }^{36}$,

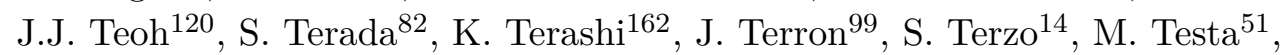
R.J. Teuscher ${ }^{166, a b}$, S.J. Thais ${ }^{182}$, N. Themistokleous ${ }^{50}$, T. Theveneaux-Pelzer ${ }^{46}$, F. Thiele ${ }^{40}$, D.W. Thomas ${ }^{94}$, J.O. Thomas ${ }^{42}$, J.P. Thomas ${ }^{21}$, E.A. Thompson ${ }^{46}$, P.D. Thompson ${ }^{21}$, E. Thomson ${ }^{136}$, E.J. Thorpe ${ }^{93}$, R.E. Ticse Torres ${ }^{53}$, V.O. Tikhomirov ${ }^{111, a h}$, Yu.A. Tikhonov ${ }^{122 b, 122 a}$, S. Timoshenko ${ }^{112}$, P. Tipton ${ }^{182}$, S. Tisserant ${ }^{102}$, K. Todome ${ }^{23 b, 23 a}$, S. Todorova-Nova ${ }^{142}$, S. Todt ${ }^{48}$, J. Tojo $^{88}$, S. Tokár ${ }^{28 a}$,

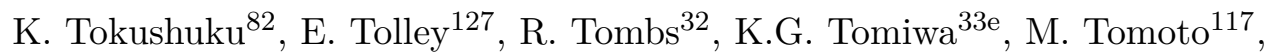
L. Tompkins ${ }^{152}$, P. Tornambe ${ }^{103}$, E. Torrence ${ }^{131}$, H. Torres ${ }^{48}$, E. Torró Pastor ${ }^{147}$,

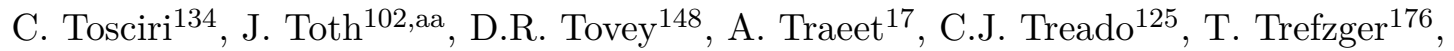
F. Tresoldi ${ }^{155}$, A. Tricoli ${ }^{29}$, I.M. Trigger ${ }^{167 a}$, S. Trincaz-Duvoid ${ }^{135}$, D.A. Trischuk ${ }^{174}$,

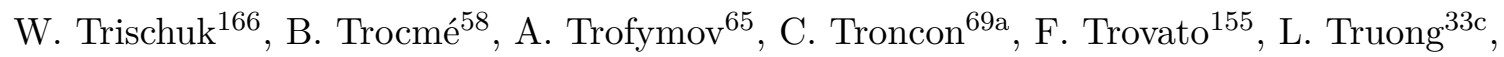
M. Trzebinski ${ }^{85}$, A. Trzupek ${ }^{85}$, F. Tsai ${ }^{46}$, J.C-L. Tseng ${ }^{134}$, P.V. Tsiareshka ${ }^{108, a e}$,

A. Tsirigotis ${ }^{161, u}$, V. Tsiskaridze ${ }^{154}$, E.G. Tskhadadze ${ }^{158 a}$, M. Tsopoulou ${ }^{161}$,

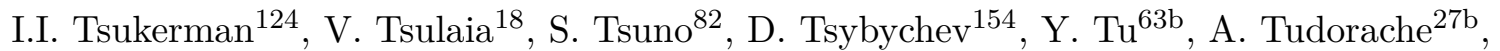
V. Tudorache ${ }^{27 b}$, T.T. Tulbure ${ }^{27 a}$, A.N. Tuna ${ }^{59}$, S. Turchikhin ${ }^{80}$, D. Turgeman ${ }^{179}$, I. Turk Cakir ${ }^{4 b, s}$, R.J. Turner ${ }^{21}$, R. Turra ${ }^{69 a}$, P.M. Tuts ${ }^{39}$, S. Tzamarias ${ }^{161}$,

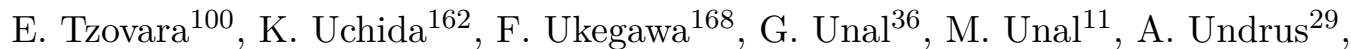

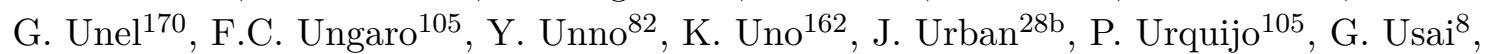
Z. Uysal ${ }^{12 \mathrm{~d}}$, V. Vacek ${ }^{141}$, B. Vachon ${ }^{104}$, K.O.H. Vadla ${ }^{133}$, T. Vafeiadis ${ }^{36}$, A. Vaidya ${ }^{95}$, C. Valderanis ${ }^{114}$, E. Valdes Santurio ${ }^{45 a, 45 b}$, M. Valente ${ }^{54}$, S. Valentinetti ${ }^{23 b, 23 a}$, A. Valero ${ }^{173}$, L. Valéry ${ }^{46}$, R.A. Vallance ${ }^{21}$, A. Vallier ${ }^{36}$, J.A. Valls Ferrer ${ }^{173}$, 
T.R. Van Daalen ${ }^{14}$, P. Van Gemmeren ${ }^{6}$, I. Van Vulpen ${ }^{120}$, M. Vanadia ${ }^{74 a, 74 b}$, W. Vandelli ${ }^{36}$, M. Vandenbroucke ${ }^{144}$, E.R. Vandewall ${ }^{129}$, A. Vaniachine ${ }^{165}$,

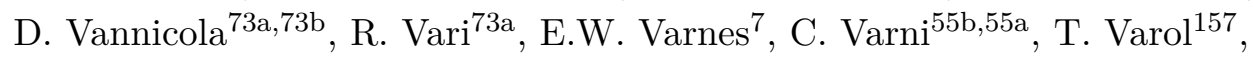
D. Varouchas ${ }^{65}$, K.E. Varvell ${ }^{156}$, M.E. Vasile ${ }^{27 b}$, G.A. Vasquez ${ }^{175}$, F. Vazeille ${ }^{38}$, D. Vazquez Furelos ${ }^{14}$, T. Vazquez Schroeder ${ }^{36}$, J. Veatch ${ }^{53}$, V. Vecchio ${ }^{101}$, M.J. Veen ${ }^{120}$,

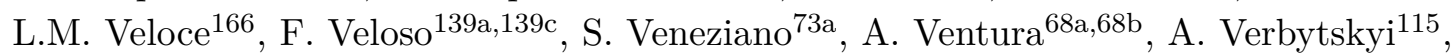
V. Vercesi ${ }^{71 a}$, M. Verducci ${ }^{72 a, 72 b}$, C.M. Vergel Infante ${ }^{79}$, C. Vergis $^{24}$, W. Verkerke $^{120}$, A.T. Vermeulen ${ }^{120}$, J.C. Vermeulen ${ }^{120}$, C. Vernieri ${ }^{152}$, M.C. Vetterli ${ }^{151, a l}$, N. Viaux Maira ${ }^{146 d}$, T. Vickey ${ }^{148}$, O.E. Vickey Boeriu ${ }^{148}$, G.H.A. Viehhauser ${ }^{134}$, L. Vigani ${ }^{61 b}$, M. Villa ${ }^{23 b, 23 a}$, M. Villaplana Perez $^{3}$, E.M. Villhauer ${ }^{50}$, E. Vilucchi ${ }^{51}$, M.G. Vincter ${ }^{34}$, G.S. Virdee ${ }^{21}$, A. Vishwakarma ${ }^{50}$, C. Vittori ${ }^{23 b, 23 a}$, I. Vivarelli ${ }^{155}$, M. Vogel ${ }^{181}$, P. Vokac ${ }^{141}$, S.E. von Buddenbrock ${ }^{33 e}$, E. Von Toerne ${ }^{24}$, V. Vorobel ${ }^{142}$, K. Vorobev ${ }^{112}$, M. Vos ${ }^{173}$, J.H. Vossebeld ${ }^{91}$, M. Vozak ${ }^{101}$, N. Vranjes ${ }^{16}$,

M. Vranjes Milosavljevic ${ }^{16}$, V. Vrba ${ }^{141}$, M. Vreeswijk ${ }^{120}$, R. Vuillermet ${ }^{36}$, I. Vukotic ${ }^{37}$, S. Wada ${ }^{168}$, P. Wagner ${ }^{24}$, W. Wagner ${ }^{181}$, J. Wagner-Kuhr ${ }^{114}$, S. Wahdan ${ }^{181}$, H. Wahlberg ${ }^{89}$, R. Wakasa ${ }^{168}$, V.M. Walbrecht ${ }^{115}$, J. Walder ${ }^{90}$, R. Walker ${ }^{114}$, S.D. Walker ${ }^{94}$, W. Walkowiak ${ }^{150}$, V. Wallangen ${ }^{45 a, 45 b}$, A.M. Wang ${ }^{59}$, A.Z. Wang ${ }^{180}$,

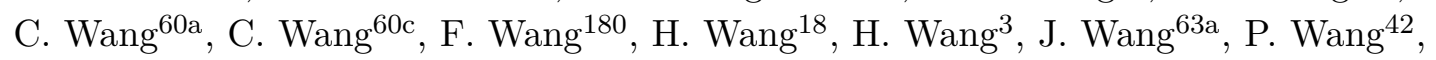
Q. Wang ${ }^{128}$, R.-J. Wang ${ }^{100}$, R. Wang ${ }^{60 a}$, R. Wang ${ }^{6}$, S.M. Wang ${ }^{157}$, W.T. Wang ${ }^{60 a}$, W. Wang ${ }^{15 c}$, W.X. Wang ${ }^{60 a}$, Y. Wang ${ }^{60 a}$, Z. Wang ${ }^{106}$, C. Wanotayaroj ${ }^{46}$, A. Warburton ${ }^{104}$, C.P. Ward $^{32}$, D.R. Wardrope ${ }^{95}$, N. Warrack ${ }^{57}$, A.T. Watson ${ }^{21}$, M.F. Watson ${ }^{21}$, G. Watts ${ }^{147}$, B.M. Waugh ${ }^{95}$, A.F. Webb ${ }^{11}$, C. Weber ${ }^{29}$, M.S. Weber ${ }^{20}$, S.A. Weber ${ }^{34}$, S.M. Weber ${ }^{61 a}$, A.R. Weidberg ${ }^{134}$, J. Weingarten ${ }^{47}$, M. Weirich ${ }^{100}$, C. Weiser ${ }^{52}$, P.S. Wells ${ }^{36}$, T. Wenaus ${ }^{29}$, B. Wendland ${ }^{47}$, T. Wengler ${ }^{36}$, S. Wenig ${ }^{36}$, N. Wermes ${ }^{24}$, M. Wessels ${ }^{61 a}$, T.D. Weston ${ }^{20}$, K. Whalen ${ }^{131}$, N.L. Whallon ${ }^{147}$, A.M. Wharton ${ }^{90}$, A.S. White ${ }^{106}$, A. White ${ }^{8}$, M.J. White ${ }^{1}$, D. Whiteson ${ }^{170}$,

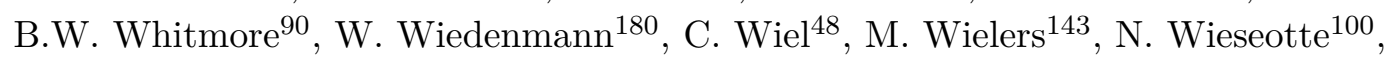
C. Wiglesworth ${ }^{40}$, L.A.M. Wiik-Fuchs ${ }^{52}$, H.G. Wilkens ${ }^{36}$, L.J. Wilkins ${ }^{94}$,

H.H. Williams ${ }^{136}$, S. Williams ${ }^{32}$, S. Willocq ${ }^{103}$, P.J. Windischhofer ${ }^{134}$, I. Wingerter-Seez ${ }^{5}$, E. Winkels ${ }^{155}$, F. Winklmeier ${ }^{131}$, B.T. Winter ${ }^{52}$, M. Wittgen ${ }^{152}$, M. Wobisch ${ }^{96}$, A. Wolf ${ }^{100}$, R. Wölker ${ }^{134}$, J. Wollrath ${ }^{52}$, M.W. Wolter ${ }^{85}$, H. Wolters ${ }^{139 a, 139 c}$, V.W.S. Wong ${ }^{174}$, N.L. Woods ${ }^{145}$, S.D. Worm ${ }^{46}$, B.K. Wosiek ${ }^{85}$, K.W. Woźniak ${ }^{85}$, K. Wraight ${ }^{57}$, S.L. $\mathrm{Wu}^{180}, \mathrm{X} . \mathrm{Wu}^{54}$, Y. Wu ${ }^{60 a}$, J. Wuerzinger ${ }^{134}$, T.R. Wyatt ${ }^{101}$, B.M. Wynne ${ }^{50}$, S. Xella ${ }^{40}$, L. Xia ${ }^{177}$, J. Xiang ${ }^{63 c}$, X. Xiao ${ }^{106}$, X. Xie ${ }^{60 a}$, I. Xiotidis ${ }^{155}$, D. $\mathrm{Xu}^{15 a}$, H. $\mathrm{Xu}^{60 \mathrm{a}}$, H. Xu ${ }^{60 \mathrm{a}}$, L. $\mathrm{Xu}^{29}$, T. $\mathrm{Xu}^{144}$, W. Xu${ }^{106}$, Z. Xu ${ }^{60 b}$, Z. Xu ${ }^{152}$, B. Yabsley ${ }^{156}$, S. Yacoob ${ }^{33 a}$, K. Yajima ${ }^{132}$, D.P. Yallup ${ }^{95}$, N. Yamaguchi ${ }^{88}$, Y. Yamaguchi ${ }^{164}$, A. Yamamoto ${ }^{82}$, M. Yamatani ${ }^{162}$, T. Yamazaki ${ }^{162}$, Y. Yamazaki $^{83}$,

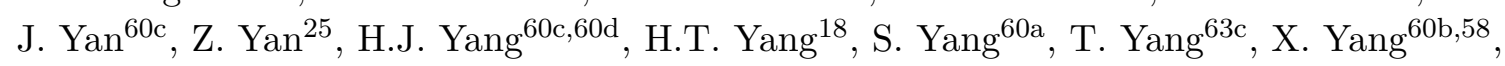
Y. Yang ${ }^{162}$, Z. Yang ${ }^{60 a}$, W-M. Yao ${ }^{18}$, Y.C. Yap ${ }^{46}$, Y. Yasu ${ }^{82}$, E. Yatsenko ${ }^{60 c}, H^{2}$ Ye $^{15 c}$, J. Ye ${ }^{42}$, S. Ye ${ }^{29}$, I. Yeletskikh ${ }^{80}$, M.R. Yexley ${ }^{90}$, E. Yigitbasi ${ }^{25}$, P. Yin ${ }^{39}$, K. Yorita ${ }^{178}$,

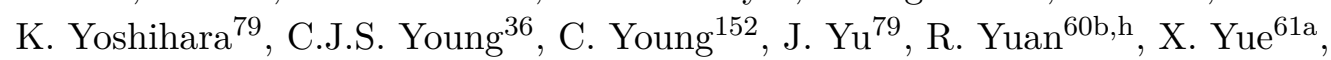
M. Zaazoua ${ }^{35 e}$, B. Zabinski ${ }^{85}$, G. Zacharis ${ }^{10}$, E. Zaffaroni ${ }^{54}$, J. Zahreddine ${ }^{135}$, A.M. Zaitsev ${ }^{123, a g}$, T. Zakareishvili ${ }^{158 b}$, N. Zakharchuk ${ }^{34}$, S. Zambito ${ }^{36}$, D. Zanzi ${ }^{36}$, 
D.R. Zaripovas ${ }^{57}$, S.V. Zeißner ${ }^{47}$, C. Zeitnitz ${ }^{181}$, G. Zemaityte ${ }^{134}$, J.C. Zeng ${ }^{172}$, O. Zenin ${ }^{123}$, T. Ženišs ${ }^{28 a}$, D. Zerwas ${ }^{65}$, M. Zgubič ${ }^{134}$, B. Zhang ${ }^{15 c}$, D.F. Zhang ${ }^{15 b}$, G. Zhang ${ }^{15 b}$, J. Zhang ${ }^{6}$, Kaili. Zhang ${ }^{15 a}$, L. Zhang ${ }^{15 c}$, L. Zhang ${ }^{60 a}$, M. Zhang ${ }^{172}$, R. Zhang ${ }^{180}$, S. Zhang ${ }^{106}$, X. Zhang ${ }^{60 c}$, X. Zhang ${ }^{60 b}$, Y. Zhang ${ }^{15 a, 15 d}$, Z. Zhang63a, Z. Zhang ${ }^{65}$, P. Zhao ${ }^{49}$, Z. Zhao ${ }^{60 a}$, A. Zhemchugov ${ }^{80}$, Z. Zheng ${ }^{106}$, D. Zhong ${ }^{172}$, B. Zhou ${ }^{106}$, C. Zhou ${ }^{180}$, H. Zhou ${ }^{7}$, M.S. Zhou ${ }^{15 a, 15 d}$, M. Zhou ${ }^{154}$, N. Zhou ${ }^{60 c}$, Y. Zhou ${ }^{7}$, C.G. Zhu ${ }^{60 b}$, C. Zhu ${ }^{15 a, 15 d}$, H.L. Zhu ${ }^{60 a}$, H. Zhu ${ }^{15 a}$, J. Zhu ${ }^{106}$, Y. Zhu ${ }^{60 a}$, X. Zhuang ${ }^{15 a}$, K. Zhukov ${ }^{111}$, V. Zhulanov ${ }^{122 b, 122 a}$, D. Zieminska ${ }^{66}$, N.I. Zimine ${ }^{80}$, S. Zimmermann ${ }^{52}$, Z. Zinonos ${ }^{115}$, M. Ziolkowski ${ }^{150}$, L. Živkovićc ${ }^{16}$, G. Zobernig ${ }^{180}$, A. Zoccoli ${ }^{23 b, 23 a}$, K. Zoch ${ }^{53}$, T.G. Zorbas ${ }^{148}$, R. Zou ${ }^{37}$, L. Zwalinski ${ }^{36}$.

1 Department of Physics, University of Adelaide, Adelaide; Australia.

2 Physics Department, SUNY Albany, Albany NY; United States of America.

3 Department of Physics, University of Alberta, Edmonton AB; Canada.

$4{ }^{(a)}$ Department of Physics, Ankara University, Ankara; ${ }^{(b)}$ Istanbul Aydin University, Application and Research Center for Advanced Studies, Istanbul; ${ }^{(c)}$ Division of Physics, TOBB University of Economics and Technology, Ankara; Turkey.

5 LAPP, Université Grenoble Alpes, Université Savoie Mont Blanc, CNRS/IN2P3, Annecy; France.

6 High Energy Physics Division, Argonne National Laboratory, Argonne IL; United States of America.

7 Department of Physics, University of Arizona, Tucson AZ; United States of America.

8 Department of Physics, University of Texas at Arlington, Arlington TX; United States of America.

9 Physics Department, National and Kapodistrian University of Athens, Athens; Greece.

10 Physics Department, National Technical University of Athens, Zografou; Greece.

11 Department of Physics, University of Texas at Austin, Austin TX; United States of America.

$12{ }^{(a)}$ Bahcesehir University, Faculty of Engineering and Natural Sciences, Istanbul; ${ }^{(b)}$ Istanbul Bilgi University, Faculty of Engineering and Natural Sciences, Istanbul; ${ }^{(c)}$ Department of Physics, Bogazici University, Istanbul $;^{(d)}$ Department of Physics Engineering, Gaziantep University, Gaziantep; Turkey.

13 Institute of Physics, Azerbaijan Academy of Sciences, Baku; Azerbaijan.

14 Institut de Física d'Altes Energies (IFAE), Barcelona Institute of Science and Technology, Barcelona; Spain.

$15{ }^{(a)}$ Institute of High Energy Physics, Chinese Academy of Sciences, Beijing; ${ }^{(b)}$ Physics Department, Tsinghua University, Beijing; ${ }^{(c)}$ Department of Physics, Nanjing University, Nanjing; ${ }^{(d)}$ University of Chinese Academy of Science (UCAS), Beijing; China.

16 Institute of Physics, University of Belgrade, Belgrade; Serbia.

17 Department for Physics and Technology, University of Bergen, Bergen; Norway.

18 Physics Division, Lawrence Berkeley National Laboratory and University of California, Berkeley CA; United States of America.

19 Institut für Physik, Humboldt Universität zu Berlin, Berlin; Germany.

20 Albert Einstein Center for Fundamental Physics and Laboratory for High Energy Physics, University of Bern, Bern; Switzerland.

21 School of Physics and Astronomy, University of Birmingham, Birmingham; United Kingdom.

${ }^{22}{ }^{(a)}$ Facultad de Ciencias y Centro de Investigaciónes, Universidad Antonio Nariño, Bogotá; ${ }^{(b)}$ Departamento de Física, Universidad Nacional de Colombia, Bogotá, Colombia; Colombia.

23 (a) INFN Bologna and Universita' di Bologna, Dipartimento di Fisica; ${ }^{(b)}$ INFN Sezione di Bologna; Italy.

24 Physikalisches Institut, Universität Bonn, Bonn; Germany.

25 Department of Physics, Boston University, Boston MA; United States of America. 
$27{ }^{(a)}$ Transilvania University of Brasov, Brasov; ${ }^{(b)}$ Horia Hulubei National Institute of Physics and Nuclear Engineering, Bucharest; ${ }^{(c)}$ Department of Physics, Alexandru Ioan Cuza University of Iasi, Iasi; ${ }^{(d)}$ National Institute for Research and Development of Isotopic and Molecular Technologies, Physics Department, Cluj-Napoca; ${ }^{(e)}$ University Politehnica Bucharest, Bucharest; ${ }^{(f)}$ West University in Timisoara, Timisoara; Romania.

28 (a) Faculty of Mathematics, Physics and Informatics, Comenius University, Bratislava; ${ }^{(b)}$ Department of Subnuclear Physics, Institute of Experimental Physics of the Slovak Academy of Sciences, Kosice; Slovak Republic.

29 Physics Department, Brookhaven National Laboratory, Upton NY; United States of America.

30 Departamento de Física, Universidad de Buenos Aires, Buenos Aires; Argentina.

31 California State University, CA; United States of America.

32 Cavendish Laboratory, University of Cambridge, Cambridge; United Kingdom.

33 (a) Department of Physics, University of Cape Town, Cape Town; ${ }^{(b)}$ iThemba Labs, Western Cape; ${ }^{(c)}$ Department of Mechanical Engineering Science, University of Johannesburg, Johannesburg; ${ }^{(d)}$ University of South Africa, Department of Physics, Pretoria; ${ }^{(e)}$ School of Physics, University of the Witwatersrand, Johannesburg; South Africa.

34 Department of Physics, Carleton University, Ottawa ON; Canada.

$35{ }^{(a)}$ Faculté des Sciences Ain Chock, Réseau Universitaire de Physique des Hautes Energies — Université Hassan II, Casablanca; ${ }^{(b)}$ Faculté des Sciences, Université Ibn-Tofail, Kénitra; ${ }^{(c)}$ Faculté des Sciences Semlalia, Université Cadi Ayyad, LPHEA-Marrakech; ${ }^{(d)}$ Faculté des Sciences, Université Mohamed Premier and LPTPM, Oujda; ${ }^{(e)}$ Faculté des sciences, Université Mohammed V, Rabat; Morocco.

36 CERN, Geneva; Switzerland.

37 Enrico Fermi Institute, University of Chicago, Chicago IL; United States of America.

38 LPC, Université Clermont Auvergne, CNRS/IN2P3, Clermont-Ferrand; France.

39 Nevis Laboratory, Columbia University, Irvington NY; United States of America.

40 Niels Bohr Institute, University of Copenhagen, Copenhagen; Denmark.

$41{ }^{(a)}$ Dipartimento di Fisica, Università della Calabria, Rende; ${ }^{(b)}$ INFN Gruppo Collegato di Cosenza, Laboratori Nazionali di Frascati; Italy.

42 Physics Department, Southern Methodist University, Dallas TX; United States of America.

43 Physics Department, University of Texas at Dallas, Richardson TX; United States of America.

44 National Centre for Scientific Research "Demokritos", Agia Paraskevi; Greece.

$45{ }^{(a)}$ Department of Physics, Stockholm University; ${ }^{(b)}$ Oskar Klein Centre, Stockholm; Sweden.

46 Deutsches Elektronen-Synchrotron DESY, Hamburg and Zeuthen; Germany.

47 Lehrstuhl für Experimentelle Physik IV, Technische Universität Dortmund, Dortmund; Germany.

48 Institut für Kern- und Teilchenphysik, Technische Universität Dresden, Dresden; Germany.

49 Department of Physics, Duke University, Durham NC; United States of America.

50 SUPA - School of Physics and Astronomy, University of Edinburgh, Edinburgh; United Kingdom.

51 INFN e Laboratori Nazionali di Frascati, Frascati; Italy.

52 Physikalisches Institut, Albert-Ludwigs-Universität Freiburg, Freiburg; Germany.

53 II. Physikalisches Institut, Georg-August-Universität Göttingen, Göttingen; Germany.

54 Département de Physique Nucléaire et Corpusculaire, Université de Genève, Genève; Switzerland.

55 (a) Dipartimento di Fisica, Università di Genova, Genova; ${ }^{(b)}$ INFN Sezione di Genova; Italy.

56 II. Physikalisches Institut, Justus-Liebig-Universität Giessen, Giessen; Germany.

57 SUPA - School of Physics and Astronomy, University of Glasgow, Glasgow; United Kingdom.

58 LPSC, Université Grenoble Alpes, CNRS/IN2P3, Grenoble INP, Grenoble; France.

59 Laboratory for Particle Physics and Cosmology, Harvard University, Cambridge MA; United States of America. 
$60{ }^{(a)}$ Department of Modern Physics and State Key Laboratory of Particle Detection and Electronics, University of Science and Technology of China, Hefei $;^{(b)}$ Institute of Frontier and Interdisciplinary Science and Key Laboratory of Particle Physics and Particle Irradiation (MOE), Shandong University, Qingdao; ${ }^{(c)}$ School of Physics and Astronomy, Shanghai Jiao Tong University, KLPPAC-MoE, SKLPPC, Shanghai; ${ }^{(d)}$ Tsung-Dao Lee Institute, Shanghai; China.

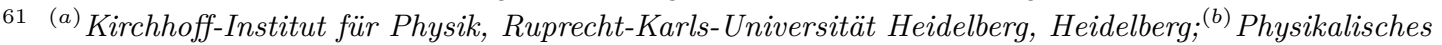
Institut, Ruprecht-Karls-Universität Heidelberg, Heidelberg; Germany.

62 Faculty of Applied Information Science, Hiroshima Institute of Technology, Hiroshima; Japan.

$63{ }^{(a)}$ Department of Physics, Chinese University of Hong Kong, Shatin, N.T., Hong Kong; ${ }^{(b)}$ Department of Physics, University of Hong Kong, Hong Kong; ${ }^{(c)}$ Department of Physics and Institute for Advanced Study, Hong Kong University of Science and Technology, Clear Water Bay, Kowloon, Hong Kong; China.

64 Department of Physics, National Tsing Hua University, Hsinchu; Taiwan.

${ }^{65}$ IJCLab, Université Paris-Saclay, CNRS/IN2P3, 91405, Orsay; France.

66 Department of Physics, Indiana University, Bloomington IN; United States of America.

$67{ }^{(a)}$ INFN Gruppo Collegato di Udine, Sezione di Trieste, Udine $;^{(b)}$ ICTP, Trieste $;^{(c)}$ Dipartimento Politecnico di Ingegneria e Architettura, Università di Udine, Udine; Italy.

$68{ }^{(a)}$ INFN Sezione di Lecce $;^{(b)}$ Dipartimento di Matematica e Fisica, Università del Salento, Lecce; Italy.

$69{ }^{(a)}$ INFN Sezione di Milano; ${ }^{(b)}$ Dipartimento di Fisica, Università di Milano, Milano; Italy.

$70{ }^{(a)}$ INFN Sezione di Napoli; ${ }^{(b)}$ Dipartimento di Fisica, Università di Napoli, Napoli; Italy.

$71{ }^{(a)}$ INFN Sezione di Pavia $;^{(b)}$ Dipartimento di Fisica, Università di Pavia, Pavia; Italy.

$72{ }^{(a)}$ INFN Sezione di Pisa; $;^{(b)}$ Dipartimento di Fisica E. Fermi, Università di Pisa, Pisa; Italy.

$73{ }^{(a)}$ INFN Sezione di Roma; ${ }^{(b)}$ Dipartimento di Fisica, Sapienza Università di Roma, Roma; Italy.

$74{ }^{(a)}$ INFN Sezione di Roma Tor Vergata $;^{(b)}$ Dipartimento di Fisica, Università di Roma Tor Vergata, Roma; Italy.

$75{ }^{(a)}$ INFN Sezione di Roma Tre ${ }^{(b)}$ Dipartimento di Matematica e Fisica, Università Roma Tre, Roma; Italy.

$76{ }^{(a)}$ INFN-TIFPA; ${ }^{(b)}$ Università degli Studi di Trento, Trento; Italy.

77 Institut für Astro- und Teilchenphysik, Leopold-Franzens-Universität, Innsbruck; Austria.

78 University of Iowa, Iowa City IA; United States of America.

79 Department of Physics and Astronomy, Iowa State University, Ames IA; United States of America.

80 Joint Institute for Nuclear Research, Dubna; Russia.

$81{ }^{(a)}$ Departamento de Engenharia Elétrica, Universidade Federal de Juiz de Fora (UFJF), Juiz de

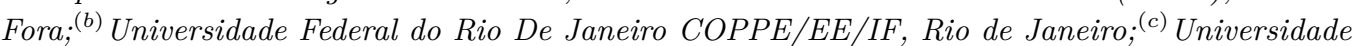
Federal de São João del Rei (UFSJ), São João del Rei; ${ }^{(d)}$ Instituto de Física, Universidade de São Paulo, São Paulo; Brazil.

82 KEK, High Energy Accelerator Research Organization, Tsukuba; Japan.

83 Graduate School of Science, Kobe University, Kobe; Japan.

$84{ }^{(a)}$ AGH University of Science and Technology, Faculty of Physics and Applied Computer Science, Krakow; ${ }^{(b)}$ Marian Smoluchowski Institute of Physics, Jagiellonian University, Krakow; Poland.

85 Institute of Nuclear Physics Polish Academy of Sciences, Krakow; Poland.

86 Faculty of Science, Kyoto University, Kyoto; Japan.

87 Kyoto University of Education, Kyoto; Japan.

88 Research Center for Advanced Particle Physics and Department of Physics, Kyushu University, Fukuoka ; Japan.

89 Instituto de Física La Plata, Universidad Nacional de La Plata and CONICET, La Plata; Argentina.

90 Physics Department, Lancaster University, Lancaster; United Kingdom.

91 Oliver Lodge Laboratory, University of Liverpool, Liverpool; United Kingdom.

92 Department of Experimental Particle Physics, Jožef Stefan Institute and Department of Physics, University of Ljubljana, Ljubljana; Slovenia. 
School of Physics and Astronomy, Queen Mary University of London, London; United Kingdom.

Department of Physics, Royal Holloway University of London, Egham; United Kingdom.

95 Department of Physics and Astronomy, University College London, London; United Kingdom.

96 Louisiana Tech University, Ruston LA; United States of America.

97 Fysiska institutionen, Lunds universitet, Lund; Sweden.

98 Centre de Calcul de l'Institut National de Physique Nucléaire et de Physique des Particules (IN2P3), Villeurbanne; France.

99 Departamento de Física Teorica C-15 and CIAFF, Universidad Autónoma de Madrid, Madrid; Spain.

100 Institut für Physik, Universität Mainz, Mainz; Germany.

101 School of Physics and Astronomy, University of Manchester, Manchester; United Kingdom.

102 CPPM, Aix-Marseille Université, CNRS/IN2P3, Marseille; France.

103 Department of Physics, University of Massachusetts, Amherst MA; United States of America.

104 Department of Physics, McGill University, Montreal QC; Canada.

105 School of Physics, University of Melbourne, Victoria; Australia.

106 Department of Physics, University of Michigan, Ann Arbor MI; United States of America.

107 Department of Physics and Astronomy, Michigan State University, East Lansing MI; United States of America.

108 B.I. Stepanov Institute of Physics, National Academy of Sciences of Belarus, Minsk; Belarus.

109 Research Institute for Nuclear Problems of Byelorussian State University, Minsk; Belarus.

110 Group of Particle Physics, University of Montreal, Montreal QC; Canada.

111 P.N. Lebedev Physical Institute of the Russian Academy of Sciences, Moscow; Russia.

112 National Research Nuclear University MEPhI, Moscow; Russia.

113 D.V. Skobeltsyn Institute of Nuclear Physics, M.V. Lomonosov Moscow State University, Moscow; Russia.

114 Fakultät für Physik, Ludwig-Maximilians-Universität München, München; Germany.

115 Max-Planck-Institut für Physik (Werner-Heisenberg-Institut), München; Germany.

116 Nagasaki Institute of Applied Science, Nagasaki; Japan.

117 Graduate School of Science and Kobayashi-Maskawa Institute, Nagoya University, Nagoya; Japan.

118 Department of Physics and Astronomy, University of New Mexico, Albuquerque NM; United States of America.

119 Institute for Mathematics, Astrophysics and Particle Physics, Radboud University Nijmegen/Nikhef, Nijmegen; Netherlands.

120 Nikhef National Institute for Subatomic Physics and University of Amsterdam, Amsterdam; Netherlands.

121 Department of Physics, Northern Illinois University, DeKalb IL; United States of America.

$122{ }^{(a)}$ Budker Institute of Nuclear Physics and NSU, SB RAS, Novosibirsk; ${ }^{(b)}$ Novosibirsk State University Novosibirsk; Russia.

123 Institute for High Energy Physics of the National Research Centre Kurchatov Institute, Protvino; Russia.

124 Institute for Theoretical and Experimental Physics named by A.I. Alikhanov of National Research Centre "Kurchatov Institute", Moscow; Russia.

125 Department of Physics, New York University, New York NY; United States of America.

126 Ochanomizu University, Otsuka, Bunkyo-ku, Tokyo; Japan.

127 Ohio State University, Columbus OH; United States of America.

128 Homer L. Dodge Department of Physics and Astronomy, University of Oklahoma, Norman OK; United States of America.

129 Department of Physics, Oklahoma State University, Stillwater OK; United States of America.

130 Palacký University, RCPTM, Joint Laboratory of Optics, Olomouc; Czech Republic.

131 Institute for Fundamental Science, University of Oregon, Eugene, OR; United States of America.

132 Graduate School of Science, Osaka University, Osaka; Japan.

133 Department of Physics, University of Oslo, Oslo; Norway. 
136 Department of Physics, University of Pennsylvania, Philadelphia PA; United States of America.

137 Konstantinov Nuclear Physics Institute of National Research Centre "Kurchatov Institute", PNPI, St. Petersburg; Russia.

138 Department of Physics and Astronomy, University of Pittsburgh, Pittsburgh PA; United States of America.

139 (a) Laboratório de Instrumentação e Física Experimental de Partículas — LIP, Lisboa; ${ }^{(b)}$ Departamento de Física, Faculdade de Ciências, Universidade de Lisboa,

Lisboa; ${ }^{(c)}$ Departamento de Física, Universidade de Coimbra, Coimbra; ${ }^{(d)}$ Centro de Física Nuclear da Universidade de Lisboa, Lisboa; ${ }^{(e)}$ Departamento de Física, Universidade do Minho,

Braga; ${ }^{(f)}$ Departamento de Física Teórica y del Cosmos, Universidad de Granada, Granada (Spain); ${ }^{(g)}$ Dep Física and CEFITEC of Faculdade de Ciências e Tecnologia, Universidade Nova de Lisboa, Caparica; ${ }^{(h)}$ Instituto Superior Técnico, Universidade de Lisboa, Lisboa; Portugal.

140 Institute of Physics of the Czech Academy of Sciences, Prague; Czech Republic.

141 Czech Technical University in Prague, Prague; Czech Republic.

142 Charles University, Faculty of Mathematics and Physics, Prague; Czech Republic.

143 Particle Physics Department, Rutherford Appleton Laboratory, Didcot; United Kingdom.

144 IRFU, CEA, Université Paris-Saclay, Gif-sur-Yvette; France.

145 Santa Cruz Institute for Particle Physics, University of California Santa Cruz, Santa Cruz CA; United States of America.

$146{ }^{(a)}$ Departamento de Física, Pontificia Universidad Católica de Chile, Santiago: ${ }^{(b)}$ Universidad $^{(a)}$ Andres Bello, Department of Physics, Santiago; ${ }^{(c)}$ Instituto de Alta Investigación, Universidad de Tarapacá; ${ }^{(d)}$ Departamento de Física, Universidad Técnica Federico Santa María, Valparaíso; Chile.

147 Department of Physics, University of Washington, Seattle WA; United States of America.

148 Department of Physics and Astronomy, University of Sheffield, Sheffield; United Kingdom.

149 Department of Physics, Shinshu University, Nagano; Japan.

150 Department Physik, Universität Siegen, Siegen; Germany.

151 Department of Physics, Simon Fraser University, Burnaby BC; Canada.

152 SLAC National Accelerator Laboratory, Stanford CA; United States of America.

153 Physics Department, Royal Institute of Technology, Stockholm; Sweden.

154 Departments of Physics and Astronomy, Stony Brook University, Stony Brook NY; United States of America.

155 Department of Physics and Astronomy, University of Sussex, Brighton; United Kingdom.

156 School of Physics, University of Sydney, Sydney; Australia.

157 Institute of Physics, Academia Sinica, Taipei; Taiwan.

$158{ }^{(a)}$ E. Andronikashvili Institute of Physics, Iv. Javakhishvili Tbilisi State University, Tbilisi; ${ }^{(b)}$ High Energy Physics Institute, Tbilisi State University, Tbilisi; Georgia.

159 Department of Physics, Technion, Israel Institute of Technology, Haifa; Israel.

160 Raymond and Beverly Sackler School of Physics and Astronomy, Tel Aviv University, Tel Aviv; Israel.

161 Department of Physics, Aristotle University of Thessaloniki, Thessaloniki; Greece.

162 International Center for Elementary Particle Physics and Department of Physics, University of Tokyo, Tokyo; Japan.

163 Graduate School of Science and Technology, Tokyo Metropolitan University, Tokyo; Japan.

164 Department of Physics, Tokyo Institute of Technology, Tokyo; Japan.

165 Tomsk State University, Tomsk; Russia.

166 Department of Physics, University of Toronto, Toronto ON; Canada.

$167{ }^{(a)}$ TRIUMF, Vancouver BC; $;^{(b)}$ Department of Physics and Astronomy, York University, Toronto ON; Canada.

168 Division of Physics and Tomonaga Center for the History of the Universe, Faculty of Pure and Applied Sciences, University of Tsukuba, Tsukuba; Japan. 
Department of Physics and Astronomy, Tufts University, Medford MA; United States of America.

Department of Physics and Astronomy, University of California Irvine, Irvine CA; United States of America.

171 Department of Physics and Astronomy, University of Uppsala, Uppsala; Sweden.

172 Department of Physics, University of Illinois, Urbana IL; United States of America.

173 Instituto de Física Corpuscular (IFIC), Centro Mixto Universidad de Valencia - CSIC, Valencia; Spain.

174 Department of Physics, University of British Columbia, Vancouver BC; Canada.

175 Department of Physics and Astronomy, University of Victoria, Victoria BC; Canada.

176 Fakultät für Physik und Astronomie, Julius-Maximilians-Universität Würzburg, Würzburg; Germany.

177 Department of Physics, University of Warwick, Coventry; United Kingdom.

178 Waseda University, Tokyo; Japan.

179 Department of Particle Physics, Weizmann Institute of Science, Rehovot; Israel.

180 Department of Physics, University of Wisconsin, Madison WI; United States of America.

181 Fakultät für Mathematik und Naturwissenschaften, Fachgruppe Physik, Bergische Universität Wuppertal, Wuppertal; Germany.

182 Department of Physics, Yale University, New Haven CT; United States of America.

a Also at Borough of Manhattan Community College, City University of New York, New York NY; United States of America.

${ }^{b}$ Also at Centro Studi e Ricerche Enrico Fermi; Italy.

c Also at CERN, Geneva; Switzerland.

${ }^{d}$ Also at CPPM, Aix-Marseille Université, CNRS/IN2P3, Marseille; France.

e Also at Département de Physique Nucléaire et Corpusculaire, Université de Genève, Genève; Switzerland.

${ }^{f}$ Also at Departament de Fisica de la Universitat Autonoma de Barcelona, Barcelona; Spain.

$g$ Also at Department of Financial and Management Engineering, University of the Aegean, Chios; Greece.

$h$ Also at Department of Physics and Astronomy, Michigan State University, East Lansing MI; United States of America.

${ }^{i}$ Also at Department of Physics and Astronomy, University of Louisville, Louisville, KY; United States of America.

${ }^{j}$ Also at Department of Physics, Ben Gurion University of the Negev, Beer Sheva; Israel.

${ }^{k}$ Also at Department of Physics, California State University, East Bay; United States of America.

${ }^{l}$ Also at Department of Physics, California State University, Fresno; United States of America.

$m$ Also at Department of Physics, California State University, Sacramento; United States of America.

${ }^{n}$ Also at Department of Physics, King's College London, London; United Kingdom.

o Also at Department of Physics, St. Petersburg State Polytechnical University, St. Petersburg; Russia.

${ }^{p}$ Also at Department of Physics, University of Fribourg, Fribourg; Switzerland.

$q$ Also at Dipartimento di Matematica, Informatica e Fisica, Università di Udine, Udine; Italy.

$r$ Also at Faculty of Physics, M.V. Lomonosov Moscow State University, Moscow; Russia.

$s$ Also at Giresun University, Faculty of Engineering, Giresun; Turkey.

${ }^{t}$ Also at Graduate School of Science, Osaka University, Osaka; Japan.

u Also at Hellenic Open University, Patras; Greece.

$v$ Also at IJCLab, Université Paris-Saclay, CNRS/IN2P3, 91405, Orsay; France.

w Also at Institucio Catalana de Recerca i Estudis Avancats, ICREA, Barcelona; Spain.

$x$ Also at Institut für Experimentalphysik, Universität Hamburg, Hamburg; Germany.

y Also at Institute for Mathematics, Astrophysics and Particle Physics, Radboud University Nijmegen/Nikhef, Nijmegen; Netherlands. 
z Also at Institute for Nuclear Research and Nuclear Energy (INRNE) of the Bulgarian Academy of Sciences, Sofia; Bulgaria.

aa Also at Institute for Particle and Nuclear Physics, Wigner Research Centre for Physics, Budapest; Hungary.

ab Also at Institute of Particle Physics (IPP); Canada.

ac Also at Institute of Physics, Azerbaijan Academy of Sciences, Baku; Azerbaijan.

ad Also at Instituto de Fisica Teorica, IFT-UAM/CSIC, Madrid; Spain.

ae Also at Joint Institute for Nuclear Research, Dubna; Russia.

af Also at Louisiana Tech University, Ruston LA; United States of America.

ag Also at Moscow Institute of Physics and Technology State University, Dolgoprudny; Russia.

ah Also at National Research Nuclear University MEPhI, Moscow; Russia.

ai Also at Physics Department, An-Najah National University, Nablus; Palestine.

aj Also at Physikalisches Institut, Albert-Ludwigs-Universität Freiburg, Freiburg; Germany.

ak Also at The City College of New York, New York NY; United States of America.

al Also at TRIUMF, Vancouver BC; Canada.

am Also at Universita di Napoli Parthenope, Napoli; Italy.

an Also at University of Chinese Academy of Sciences (UCAS), Beijing; China.

* Deceased 